\title{
Organic Light-Emitting Devices with High External Quantum Efficiency and Operational Stability Based on Highly Phosphorescent Cyclometalating Rhodium(III) Complexes
}

Fangfang Wei, ${ }^{a \dagger}$ Zhiyuan Qian, ${ }^{a \dagger}$ Shiu-Lun Lai, ${ }^{b}$ Shunan Zhao, ${ }^{\mathrm{a}}$ Mei-Yee Chan, ${ }^{\mathrm{b} *}$ and Keith Man-Chung Wong*a

${ }^{a}$ Department of Chemistry, Southern University of Science and Technology, 1088 Xueyuan Blvd., Shenzhen 518055, P.R. China.

b Institute of Molecular Functional Materials (Areas of Excellence Scheme, University Grants Committee, Hong Kong) and Department of Chemistry, The University of Hong Kong, Pokfulam Road, Hong Kong, P. R. China.

\section{Supporting Information}



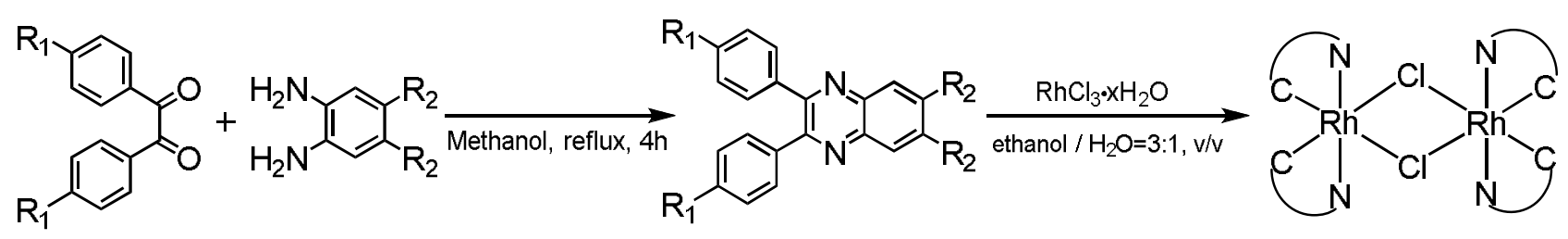

$$
\begin{array}{ll}
\mathrm{R}_{1}=\mathrm{CH}_{3}, \mathrm{R}_{2}=\mathrm{H} & \left(\mathbf{L}_{1}\right) \\
\mathrm{R}_{1}=\mathrm{H}, \mathrm{R}_{2}=\mathrm{CH}_{3} & \left(\mathrm{~L}_{2}\right) \\
\mathrm{R}_{1}=\mathrm{H}, \mathrm{R}_{2}=\mathrm{F} & \left(\mathrm{L}_{3}\right) \\
\mathrm{R}_{1}=\mathrm{CH}_{3}, \mathrm{R}_{2}=\mathrm{F} & \left(\mathbf{L}_{4}\right) \\
\mathrm{R}_{1}=\mathrm{H}, \mathrm{R}_{2}=\mathrm{H} & \left(\mathrm{L}_{5}\right)
\end{array}
$$

$\left[\mathrm{Rh}-\left(\mathrm{C}^{\wedge} \mathrm{N}\right)_{2}-\mathrm{Cl}_{2}\right]_{2}$ $\mathrm{C}^{\wedge} \mathrm{N}=\mathrm{L}_{1}-\mathrm{L}_{5}$

Scheme $\mathbf{S} 1$. General synthetic procedures of $\mathrm{L}_{\mathbf{1}}-\mathrm{L}_{4}$ and $\left[\mathrm{Rh}\left(\mathrm{C}^{\wedge} N\right)_{2} \mathrm{Cl}\right]_{2}$. 
Table S1. Crystal and structure determination data of rhodium(III) 1-6.

\begin{tabular}{|c|c|c|c|c|c|c|}
\hline & 1 & 2 & 3 & 4 & 5 & 6 \\
\hline \multirow{3}{*}{ empirical formula } & $\mathrm{C}_{61} \mathrm{H}_{48} \mathrm{Cl}_{5} \mathrm{~N}_{4} \mathrm{O}$ & $\mathrm{C}_{61} \mathrm{H}_{49} \mathrm{Cl}_{4} \mathrm{~N}_{4} \mathrm{O}_{2}$ & $\mathrm{C}_{113} \mathrm{H}_{66} \mathrm{Cl}_{6} \mathrm{~N}_{8} \mathrm{O}$ & $\mathrm{C}_{60} \mathrm{H}_{43} \mathrm{Cl}_{2} \mathrm{~N}_{4} \mathrm{O}_{2} \mathrm{~F}$ & \multirow{3}{*}{$\mathrm{C}_{51} \mathrm{H}_{42} \mathrm{Cl}_{2} \mathrm{~N}_{5} \mathrm{O}_{3} \mathrm{Rh}$} & \multirow{3}{*}{$\mathrm{C}_{48} \mathrm{H}_{33} \mathrm{~N}_{6} \mathrm{O}_{3} \mathrm{Rh}$} \\
\hline & & & & & & \\
\hline & ${ }_{2} \mathrm{Rh}$ & $\mathrm{Rh}$ & ${ }_{4} \mathrm{~F}_{8} \mathrm{Rh}_{2}$ & ${ }_{4} \mathrm{Rh}$ & & \\
\hline formula weight & 1149.19 & 1114.75 & 2170.25 & 1101.79 & 946.70 & 844.71 \\
\hline temperature, $\mathrm{K}$ & 100.01 & 112.46 & 99.98 & 100.04 & $150(2)$ & 100 \\
\hline Wavelength, $\AA$ & 0.71073 & 0.71073 & 0.71073 & 1.54178 & 0.71057 & 1.54178 \\
\hline Cryst syst & Monoclinic & Triclinic & Triclinic & Monoclinic & Monoclinic & Triclinic \\
\hline Space group & $\mathrm{P} 121 / \mathrm{C} 1$ & $\mathrm{P}-1$ & $\mathrm{P}-1$ & $\mathrm{P} 121 / \mathrm{C} 1$ & $\mathrm{P} 2(1) / \mathrm{n}$ & $\mathrm{P}-1$ \\
\hline $\mathrm{a}, \AA$ & $11.6284(7)$ & $11.5378(10)$ & $11.6218(4)$ & $11.0998(3)$ & $14.7105(8)$ & $9.8456(3)$ \\
\hline $\mathrm{b}, \AA$ & $29.2329(14)$ & $14.4955(13)$ & $12.6773(5)$ & $36.9703(10)$ & $15.7362(9)$ & $13.5298(4)$ \\
\hline $\mathrm{c}, \AA$ & $15.0903(8)$ & $15.9053(14)$ & $17.7544(7)$ & $12.8226(3)$ & $19.4856(11)$ & $14.5378(4)$ \\
\hline$\alpha, \operatorname{deg}$ & 90 & $92.581(3)$ & $72.7800(10)$ & 90 & 90 & 90.6900 \\
\hline$\beta, \operatorname{deg}$ & $94.564(2)$ & $99.037(3)$ & $87.6370(10)$ & $110.917(2)$ & $100.7088(17)$ & 103.2950 \\
\hline$\gamma, \operatorname{deg}$ & 90 & $99.170(3)$ & $68.1660(10)$ & 90 & 90 & 90.6470 \\
\hline volume, $\AA^{3}$ & $5113.4(5)$ & $2586.8(4)$ & $2312.32(15)$ & $4915.2(2)$ & 4432.1(4) & 1884.33(10) \\
\hline $\mathrm{Z}$ & 4 & 2 & 1 & 4 & 4 & 2 \\
\hline Density (calcd), g & 1.493 & 1.431 & 1.559 & 1.489 & 1.419 & 1.489 \\
\hline Cryst size, & $0.21 \times 0.19 \times 0$ & $0.21 \times 0.19 \times 0.17$ & $0.21 \times 0.19 \times 0.17$ & $0.21 \times 0.19 \times 0.17$ & $0.21 \times 0.19 \times 0.17$ & $0.21 \times 0.19 \times 0.17$ \\
\hline \multirow[t]{2}{*}{$\mathrm{mm} X \mathrm{~mm} X \mathrm{~mm}$} & 17 & & & & & \\
\hline & $-15 \leq h \leq 13$ & $-14 \leq \mathrm{h} \leq 15$ & $-15 \leq h \leq 15$ & $-13 \leq h \leq 13$ & $-19 \leq \mathrm{h} \leq 19$ & $-11 \leq \mathrm{h} \leq 11$ \\
\hline \multirow[t]{3}{*}{ index ranges } & $-37 \leq \mathrm{k} \leq 37$ & $-18 \leq \mathrm{k} \leq 18$ & $-16 \leq \mathrm{k} \leq 16$ & $-42 \leq \mathrm{k} \leq 43$ & $-20 \leq \mathrm{k} \leq 20$ & $-15 \leq \mathrm{k} \leq 15$ \\
\hline & $-16 \leq 1 \leq 19$ & $-20 \leq 1 \leq 20$ & $-23 \leq 1 \leq 23$ & $-15 \leq 1 \leq 15$ & $-25 \leq 1 \leq 25$ & $-17 \leq 1 \leq 16$ \\
\hline & $53814 / 11735$ & 44283 / 11943 & $42940 / 10620$ & & & \\
\hline reflns & & & & 44035 / 8340 & $96497 / 10235$ & 30405 / 6405 \\
\hline \multirow[t]{2}{*}{ collected/unique } & {$[\mathrm{R}$ (int) $=$} & {$[\mathrm{R}($ int $)=$} & {$[\mathrm{R}($ int $)=$} & {$[R($ int $)=0.069]$} & {$[\mathrm{R}(\mathrm{int})=0.0399]$} & {$[\mathrm{R}(\mathrm{int})=0.0364]$} \\
\hline & 0.0984] & $0.0657]$ & $0.0463]$ & & & \\
\hline
\end{tabular}




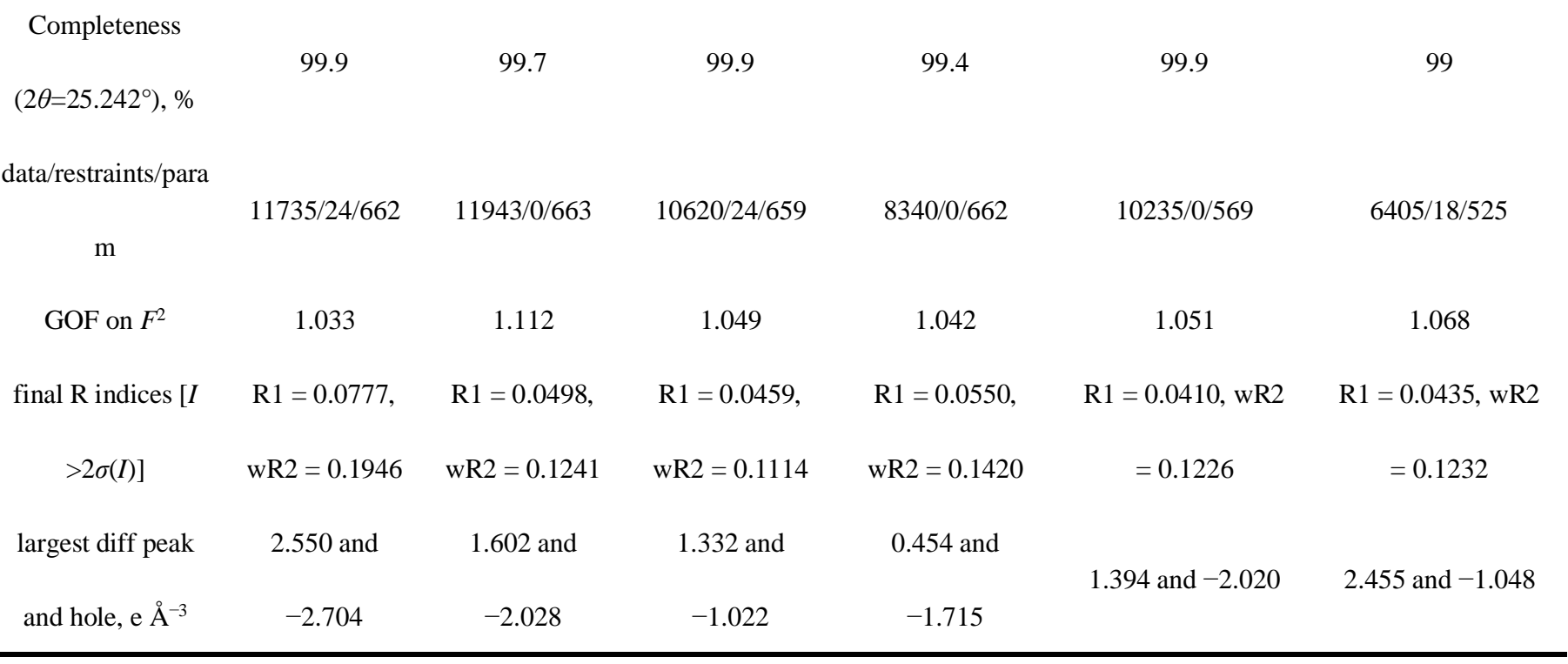


Table S2. Selected bond lengths $[\AA]$ and angles [deg] of 1-6.

\begin{tabular}{|c|c|c|c|}
\hline \multicolumn{4}{|c|}{1 Bond Lengths $(\AA)$} \\
\hline $\mathrm{Rh}(1)-\mathrm{O}(2)$ & $2.139(3)$ & $\operatorname{Rh}(1)-\mathrm{N}(1)$ & $2.061(4)$ \\
\hline $\mathrm{Rh}(1)-\mathrm{O}(1)$ & $2.166(4)$ & $\mathrm{Rh}(1)-\mathrm{C}(9)$ & $1.973(5)$ \\
\hline $\operatorname{Rh}(1)-N(3)$ & $2.039(4)$ & $\mathrm{Rh}(1)-\mathrm{C}(31)$ & $1.965(5)$ \\
\hline \multicolumn{4}{|c|}{1 Bond Angles (deg) } \\
\hline $\mathrm{O}(2)-\mathrm{Rh}(1)-\mathrm{O}(1)$ & $86.17(13)$ & $\mathrm{C}(9)-\mathrm{Rh}(1)-\mathrm{N}(3)$ & $95.96(18)$ \\
\hline $\mathrm{N}(3)-\mathrm{Rh}(1)-\mathrm{O}(2)$ & $84.34(15)$ & $\mathrm{C}(9)-\mathrm{Rh}(1)-\mathrm{N}(1)$ & $80.43(18)$ \\
\hline $\mathrm{N}(3)-\mathrm{Rh}(1)-\mathrm{O}(1)$ & $97.34(15)$ & $\mathrm{C}(31)-\mathrm{Rh}(1)-\mathrm{O}(2)$ & $86.64(16)$ \\
\hline $\mathrm{N}(3)-\mathrm{Rh}(1)-\mathrm{N}(1)$ & $176.27(16)$ & $\mathrm{C}(31)-\mathrm{Rh}(1)-\mathrm{O}(1)$ & $172.73(16)$ \\
\hline $\mathrm{N}(1)-\mathrm{Rh}(1)-\mathrm{O}(2)$ & $99.28(15)$ & $\mathrm{C}(31)-\mathrm{Rh}(1)-\mathrm{N}(3)$ & $80.88(18)$ \\
\hline $\mathrm{N}(1)-\mathrm{Rh}(1)-\mathrm{O}(1)$ & $83.85(15)$ & $\mathrm{C}(31)-\mathrm{Rh}(1)-\mathrm{N}(1)$ & $98.37(18)$ \\
\hline $\mathrm{C}(9)-\mathrm{Rh}(1)-\mathrm{O}(2)$ & $179.00(18)$ & $\mathrm{C}(31)-\mathrm{Rh}(1)-\mathrm{C}(9)$ & $94.36(19)$ \\
\hline $\mathrm{C}(9)-\mathrm{Rh}(1)-\mathrm{O}(1)$ & $92.84(17)$ & & \\
\hline \multicolumn{4}{|c|}{2 Bond Lengths $(\AA)$} \\
\hline $\mathrm{Rh}(1)-\mathrm{O}(2)$ & $2.154(2)$ & $\operatorname{Rh}(1)-\mathrm{N}(3)$ & $2.071(2)$ \\
\hline $\mathrm{Rh}(1)-\mathrm{O}(1)$ & $2.167(2)$ & $\mathrm{Rh}(1)-\mathrm{C}(1)$ & $1.967(3)$ \\
\hline $\mathrm{Rh}(1)-\mathrm{N}(1)$ & $2.053(2)$ & $\mathrm{Rh}(1)-\mathrm{C}(23)$ & $1.966(3)$ \\
\hline \multicolumn{4}{|c|}{2 Bond Angles (deg) } \\
\hline $\mathrm{O}(2)-\mathrm{Rh}(1)-\mathrm{O}(1)$ & $84.73(8)$ & $\mathrm{C}(1)-\mathrm{Rh}(1)-\mathrm{N}(1)$ & $80.53(11)$ \\
\hline $\mathrm{N}(1)-\mathrm{Rh}(1)-\mathrm{O}(2)$ & $82.19(9)$ & $\mathrm{C}(1)-\mathrm{Rh}(1)-\mathrm{N}(3)$ & $97.06(11)$ \\
\hline $\mathrm{N}(1)-\mathrm{Rh}(1)-\mathrm{O}(1)$ & $101.94(9)$ & $\mathrm{C}(23)-\mathrm{Rh}(1)-\mathrm{O}(2)$ & $175.88(10)$ \\
\hline $\mathrm{N}(1)-\mathrm{Rh}(1)-\mathrm{N}(3)$ & $173.55(9)$ & $\mathrm{C}(23)-\mathrm{Rh}(1)-\mathrm{O}(1)$ & $95.67(10)$ \\
\hline $\mathrm{N}(3)-\mathrm{Rh}(1)-\mathrm{O}(2)$ & $103.78(9)$ & $\mathrm{C}(23)-\mathrm{Rh}(1)-\mathrm{N}(1)$ & $93.72(11)$ \\
\hline $\mathrm{N}(3)-\mathrm{Rh}(1)-\mathrm{O}(1)$ & $81.25(9)$ & $\mathrm{C}(23)-\mathrm{Rh}(1)-\mathrm{N}(3)$ & $80.33(11)$ \\
\hline $\mathrm{C}(1)-\mathrm{Rh}(1)-\mathrm{O}(2)$ & $88.23(10)$ & $\mathrm{C}(23)-\mathrm{Rh}(1)-\mathrm{C}(1)$ & $91.60(12)$ \\
\hline $\mathrm{C}(1)-\mathrm{Rh}(1)-\mathrm{O}(1)$ & $172.13(10)$ & & \\
\hline
\end{tabular}

3 Bond Lengths ( $)$ 


\begin{tabular}{llll}
$\mathrm{Rh}(1)-\mathrm{O}(2)$ & $2.148(2)$ & $\mathrm{Rh}(1)-\mathrm{N}(3)$ & $2.042(2)$ \\
$\mathrm{Rh}(1)-\mathrm{O}(1)$ & $2.138(2)$ & $\mathrm{Rh}(1)-\mathrm{C}(1)$ & $1.974(3)$ \\
$\mathrm{Rh}(1)-\mathrm{N}(1)$ & $2.055(2)$ & $\mathrm{Rh}(1)-\mathrm{C}(21)$ & $1.964(3)$ \\
\hline \multicolumn{4}{c}{3 Bond Angles $(\mathrm{deg})$} \\
$\mathrm{O}(1)-\mathrm{Rh}(1)-\mathrm{O}(2)$ & $85.99(8)$ & $\mathrm{C}(1)-\mathrm{Rh}(1)-\mathrm{N}(1)$ & $80.59(11)$ \\
$\mathrm{N}(1)-\mathrm{Rh}(1)-\mathrm{O}(2)$ & $99.44(9)$ & $\mathrm{C}(1)-\mathrm{Rh}(1)-\mathrm{N}(3)$ & $96.86(11)$ \\
$\mathrm{N}(1)-\mathrm{Rh}(1)-\mathrm{O}(1)$ & $85.24(9)$ & $\mathrm{C}(21)-\mathrm{Rh}(1)-\mathrm{O}(2)$ & $89.13(10)$ \\
$\mathrm{N}(3)-\mathrm{Rh}(1)-\mathrm{O}(2)$ & $83.31(9)$ & $\mathrm{C}(21)-\mathrm{Rh}(1)-\mathrm{O}(1)$ & $174.96(10)$ \\
$\mathrm{N}(3)-\mathrm{Rh}(1)-\mathrm{O}(1)$ & $97.56(9)$ & $\mathrm{C}(21)-\mathrm{Rh}(1)-\mathrm{N}(1)$ & $96.80(11)$ \\
$\mathrm{N}(3)-\mathrm{Rh}(1)-\mathrm{N}(1)$ & $176.24(10)$ & $\mathrm{C}(21)-\mathrm{Rh}(1)-\mathrm{N}(3)$ & $80.62(11)$ \\
$\mathrm{C}(1)-\mathrm{Rh}(1)-\mathrm{O}(2)$ & $175.71(10)$ & $\mathrm{C}(21)-\mathrm{Rh}(1)-\mathrm{C}(1)$ & $95.13(12)$ \\
$\mathrm{C}(1)-\mathrm{Rh}(1)-\mathrm{O}(1)$ & $89.74(10)$ & & \\
\hline
\end{tabular}

4 Bond Lengths $(\AA)$

\begin{tabular}{llll}
$\mathrm{Rh}(1)-\mathrm{O}(1)$ & $2.149(4)$ & $\mathrm{Rh}(1)-\mathrm{N}(1)$ & $2.055(4)$ \\
$\mathrm{Rh}(1)-\mathrm{O}(2)$ & $2.156(3)$ & $\mathrm{Rh}(1)-\mathrm{C}(1)$ & $1.959(5)$ \\
$\mathrm{Rh}(1)-\mathrm{N}(3)$ & $2.055(4)$ & $\mathrm{Rh}(1)-\mathrm{C}(23)$ & $1.966(5)$ \\
\hline
\end{tabular}

4 Bond Angles (deg)

\begin{tabular}{llll}
$\mathrm{O}(1)-\mathrm{Rh}(1)-\mathrm{O}(2)$ & $85.15(14)$ & $\mathrm{C}(1)-\mathrm{Rh}(1)-\mathrm{N}(3)$ & $95.76(18)$ \\
$\mathrm{N}(3)-\mathrm{Rh}(1)-\mathrm{O}(1)$ & $99.89(15)$ & $\mathrm{C}(1)-\mathrm{Rh}(1)-\mathrm{N}(1)$ & $80.84(18)$ \\
$\mathrm{N}(3)-\mathrm{Rh}(1)-\mathrm{O}(2)$ & $84.05(15)$ & $\mathrm{C}(1)-\mathrm{Rh}(1)-\mathrm{C}(23)$ & $92.0(2)$ \\
$\mathrm{N}(1)-\mathrm{Rh}(1)-\mathrm{O}(1)$ & $84.04(15)$ & $\mathrm{C}(23)-\mathrm{Rh}(1)-\mathrm{O}(1)$ & $177.11(16)$ \\
$\mathrm{N}(1)-\mathrm{Rh}(1)-\mathrm{O}(2)$ & $99.62(15)$ & $\mathrm{C}(23)-\mathrm{Rh}(1)-\mathrm{O}(2)$ & $92.16(17)$ \\
$\mathrm{N}(1)-\mathrm{Rh}(1)-\mathrm{N}(3)$ & $174.88(16)$ & $\mathrm{C}(23)-\mathrm{Rh}(1)-\mathrm{N}(3)$ & $80.85(18)$ \\
$\mathrm{C}(1)-\mathrm{Rh}(1)-\mathrm{O}(1)$ & $90.71(17)$ & $\mathrm{C}(23)-\mathrm{Rh}(1)-\mathrm{N}(1)$ & $95.39(18)$ \\
$\mathrm{C}(1)-\mathrm{Rh}(1)-\mathrm{O}(2)$ & $175.75(17)$ & & \\
\hline
\end{tabular}

5 Bond Lengths $(\AA)$

$\begin{array}{llll}\mathrm{Rh}(1)-\mathrm{C}(1) & 1.973(3) & \mathrm{Rh}(1)-\mathrm{N}(1) & 2.064(2) \\ \mathrm{Rh}(1)-\mathrm{C}(21) & 1.976(3) & \mathrm{Rh}(1)-\mathrm{O}(1) & 2.1654(18)\end{array}$




\begin{tabular}{|c|c|c|c|}
\hline $\mathrm{Rh}(1)-\mathrm{N}(3)$ & $2.052(2)$ & $\operatorname{Rh}(1)-\mathrm{N}(5)$ & $2.172(2)$ \\
\hline \multicolumn{4}{|c|}{5 Bond Angles (deg) } \\
\hline $\mathrm{C}(1)-\mathrm{Rh}(1)-\mathrm{C}(21)$ & $93.77(11)$ & $\mathrm{N}(3)-\mathrm{Rh}(1)-\mathrm{O}(1)$ & $80.61(8)$ \\
\hline $\mathrm{C}(1)-\mathrm{Rh}(1)-\mathrm{N}(3)$ & $97.49(10)$ & $\mathrm{N}(1)-\mathrm{Rh}(1)-\mathrm{O}(1)$ & $102.17(8)$ \\
\hline $\mathrm{C}(21)-\mathrm{Rh}(1)-\mathrm{N}(3)$ & $80.48(10)$ & $\mathrm{C}(1)-\mathrm{Rh}(1)-\mathrm{N}(5)$ & $99.36(10)$ \\
\hline $\mathrm{C}(1)-\mathrm{Rh}(1)-\mathrm{N}(1)$ & $79.90(10)$ & $\mathrm{C}(21)-\mathrm{Rh}(1)-\mathrm{N}(5)$ & $166.80(10)$ \\
\hline $\mathrm{C}(21)-\mathrm{Rh}(1)-\mathrm{N}(1)$ & $97.50(10)$ & $\mathrm{N}(3)-\mathrm{Rh}(1)-\mathrm{N}(5)$ & $99.08(8)$ \\
\hline $\mathrm{N}(3)-\mathrm{Rh}(1)-\mathrm{N}(1)$ & $176.63(8)$ & $\mathrm{N}(1)-\mathrm{Rh}(1)-\mathrm{N}(5)$ & $83.50(8)$ \\
\hline $\mathrm{C}(1)-\mathrm{Rh}(1)-\mathrm{O}(1)$ & $174.80(9)$ & $\mathrm{O}(1)-\mathrm{Rh}(1)-\mathrm{N}(5)$ & $76.26(8)$ \\
\hline $\mathrm{C}(21)-\mathrm{Rh}(1)-\mathrm{O}(1)$ & \multicolumn{3}{|l|}{$90.70(9)$} \\
\hline \multicolumn{4}{|c|}{6 Bond Lengths $(\AA)$} \\
\hline $\operatorname{Rh}(1)-\mathrm{O}(1)$ & $2.188(3)$ & $\mathrm{Rh}(1)-\mathrm{N}(5)$ & $2.194(3)$ \\
\hline $\mathrm{Rh}(1)-\mathrm{N}(1)$ & $2.040(3)$ & $\mathrm{Rh}(1)-\mathrm{C}(1)$ & $1.970(4)$ \\
\hline $\operatorname{Rh}(1)-\mathrm{N}(3)$ & $2.079(3)$ & $\mathrm{Rh}(1)-\mathrm{C}(21)$ & $1.987(4)$ \\
\hline \multicolumn{4}{|c|}{6 Bond Angles (deg) } \\
\hline $\mathrm{O}(1)-\mathrm{Rh}(1)-\mathrm{N}(5)$ & $76.73(11)$ & $\mathrm{C}(1)-\mathrm{Rh}(1)-\mathrm{N}(3)$ & $99.43(15)$ \\
\hline $\mathrm{N}(1)-\mathrm{Rh}(1)-\mathrm{O}(1)$ & $99.82(11)$ & $\mathrm{C}(1)-\mathrm{Rh}(1)-\mathrm{N}(5)$ & $94.27(14)$ \\
\hline $\mathrm{N}(1)-\mathrm{Rh}(1)-\mathrm{N}(3)$ & $173.95(12)$ & $\mathrm{C}(1)-\mathrm{Rh}(1)-\mathrm{C}(21)$ & $94.61(15)$ \\
\hline $\mathrm{N}(1)-\mathrm{Rh}(1)-\mathrm{N}(5)$ & $85.55(12)$ & $\mathrm{C}(21)-\mathrm{Rh}(1)-\mathrm{O}(1)$ & $94.42(13)$ \\
\hline $\mathrm{N}(3)-\mathrm{Rh}(1)-\mathrm{O}(1)$ & $81.56(11)$ & $\mathrm{C}(21)-\mathrm{Rh}(1)-\mathrm{N}(1)$ & $93.82(14)$ \\
\hline $\mathrm{N}(3)-\mathrm{Rh}(1)-\mathrm{N}(5)$ & $100.49(12)$ & $\mathrm{C}(21)-\mathrm{Rh}(1)-\mathrm{N}(3)$ & $80.18(14)$ \\
\hline $\mathrm{C}(1)-\mathrm{Rh}(1)-\mathrm{O}(1)$ & $170.95(13)$ & $\mathrm{C}(21)-\mathrm{Rh}(1)-\mathrm{N}(5)$ & $170.85(13)$ \\
\hline $\mathrm{C}(1)-\mathrm{Rh}(1)-\mathrm{N}(1)$ & $80.15(15)$ & & \\
\hline
\end{tabular}


Table S3. Key characteristics of solution-processed devices made with 2, 3, and $\mathbf{5}$.

\begin{tabular}{cccccc}
\hline Complex & $\begin{array}{c}\text { Max. } \mathrm{CE}^{\mathrm{a}}, \\
\mathrm{cd} \mathrm{A}^{-1}\end{array}$ & $\begin{array}{c}\text { Max. } \mathrm{PE}^{\mathrm{b}}, \\
\mathrm{lm} \mathrm{W^{-1 }}\end{array}$ & $\begin{array}{c}\text { Max. EQE, } \\
\%\end{array}$ & $\begin{array}{c}\lambda_{\max }{ }^{\mathrm{c}}, \mathrm{nm} \\
(\mathrm{FWHM}, \mathrm{nm})\end{array}$ & $\mathrm{CIEx}^{\mathrm{d}} y^{\mathrm{d}}$ \\
\hline $\mathbf{2}$ & 1.3 & 0.3 & 1.3 & $600(88)$ & $0.63,0.37$ \\
$\mathbf{3}$ & 4.5 & 1.4 & 2.8 & $600(78)$ & $0.62,0.38$ \\
$\mathbf{5}$ & 10.3 & 2.6 & 6.0 & $596(83)$ & $0.61,0.39$ \\
\hline
\end{tabular}

${ }^{\mathrm{a}} \mathrm{CE}$ represents current efficiency.

${ }^{\mathrm{b}} \mathrm{PE}$ represents power efficiency.

${ }^{\mathrm{c}} \lambda_{\max }$ represents peak maximum.

${ }^{\mathrm{d}} \mathrm{CIE}$ coordinates taken at luminance of $100 \mathrm{~cd} \mathrm{~m}^{-2}$. 
Table S4. Device lifetime data of the vacuum-deposited devices based on $\mathbf{2}$ and $\mathbf{3 .}$

\begin{tabular}{ccccc}
\hline Complex & $\begin{array}{c}\text { Initial } \\
\text { brightness, } \\
\mathrm{cd} \mathrm{m}^{-2}\end{array}$ & $\mathrm{LT}_{50}{ }^{\mathrm{a}}$ at $\mathrm{L}_{0}$ & $\begin{array}{c}\mathrm{LT}_{50}{ }^{\mathrm{b}} \text { at } \\
1000 \mathrm{~cd} \mathrm{~m}^{-2}\end{array}$ & $\begin{array}{c}\mathrm{LT}_{50} \\
\text { at } 100 \mathrm{~cd} \mathrm{~m}^{-2}\end{array}$ \\
\hline $\mathbf{2}$ & 1115 & 98.2 & 118 & 5925 \\
$\mathbf{3}$ & 1653 & 147.1 & 346 & 17322 \\
\hline
\end{tabular}

${ }^{\mathrm{a}} \mathrm{LT}_{50}$ is defined as the operational lifetime at $50 \%$ of initial luminance.

${ }^{\mathrm{b}} \mathrm{L}_{0}$ is defined as the initial luminance. 


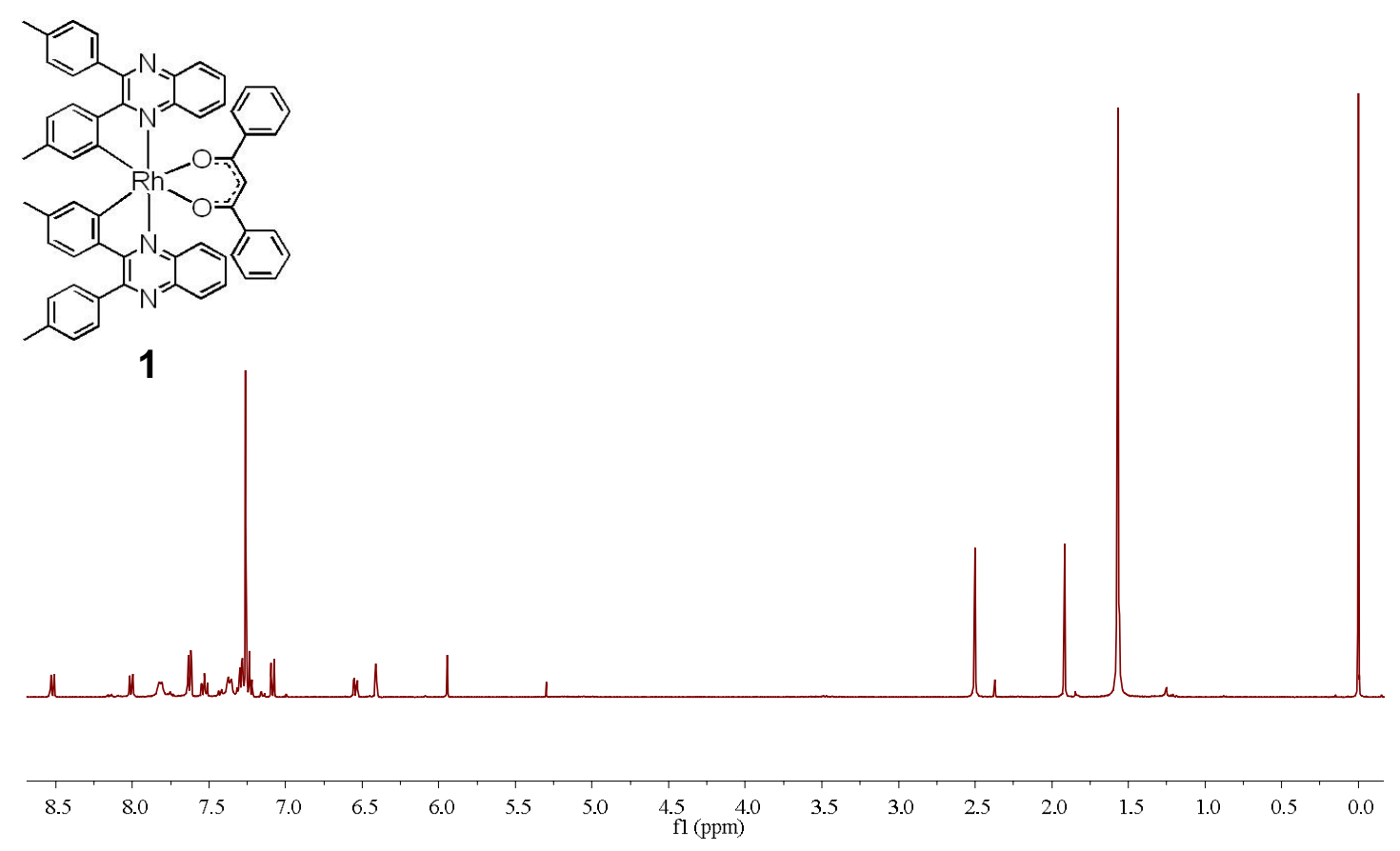

Figure S1. ${ }^{1} \mathrm{H}$ NMR spectrum of 1 in $\mathrm{CDCl}_{3}$ at $298 \mathrm{~K}$.

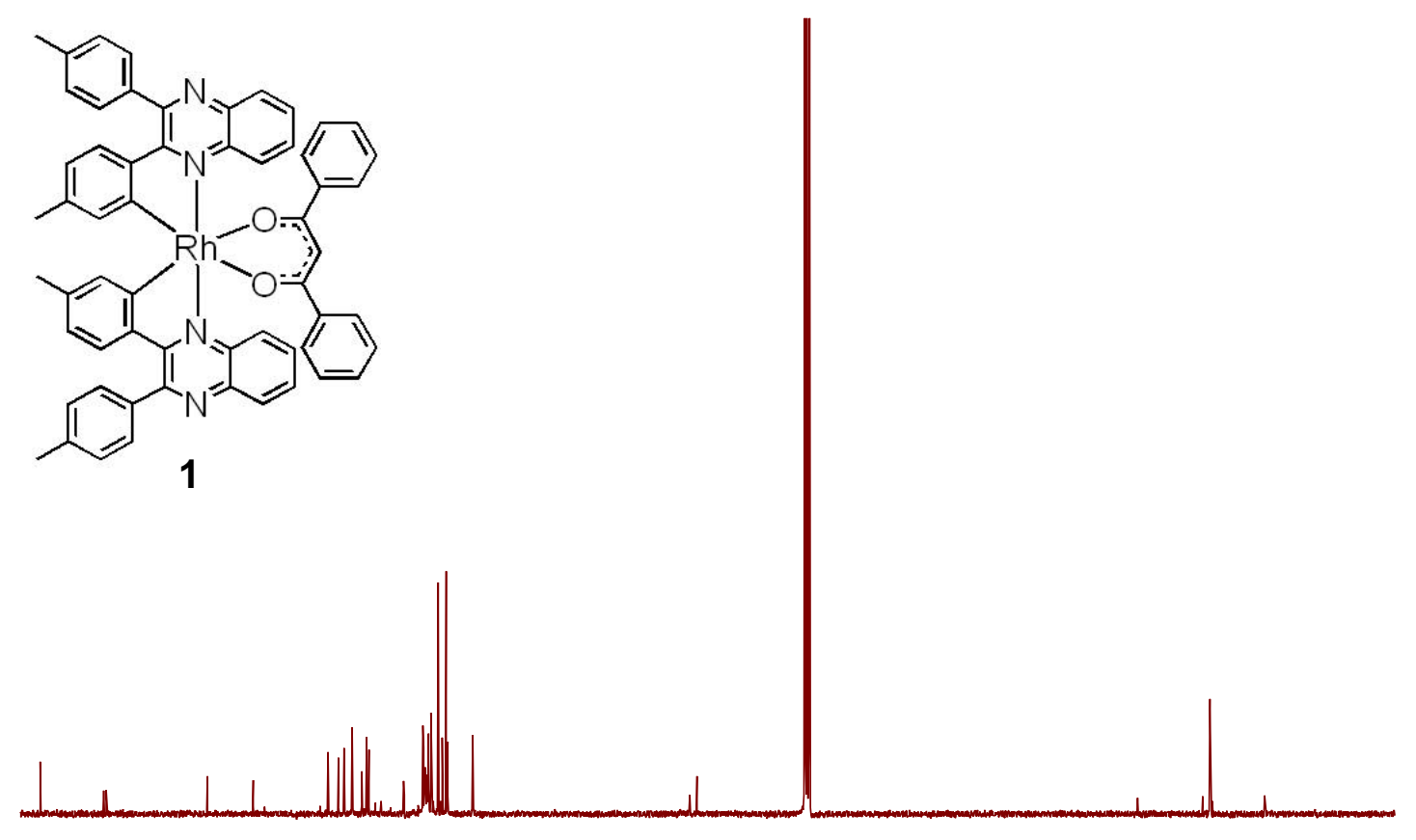

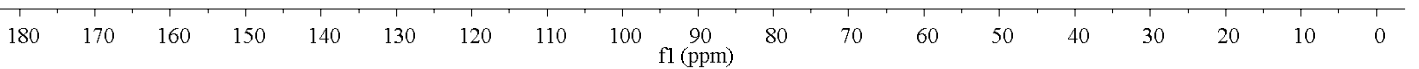

Figure S2. ${ }^{13} \mathrm{C}$ NMR spectrum of $\mathbf{1}$ in $\mathrm{CDCl}_{3}$ at $298 \mathrm{~K}$. 

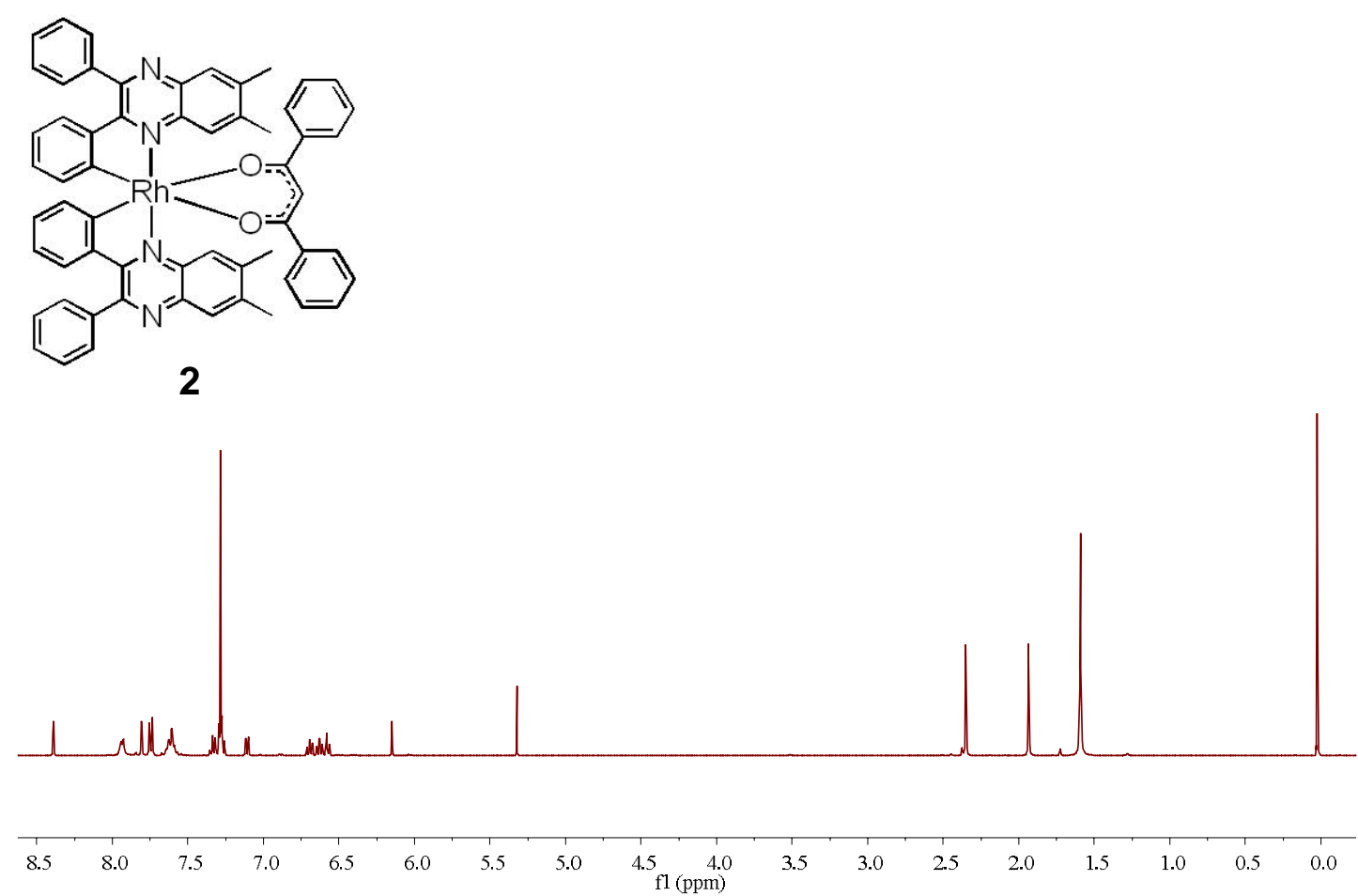

Figure S3. ${ }^{1} \mathrm{H}$ NMR spectrum of 2 in $\mathrm{CDCl}_{3}$ at $298 \mathrm{~K}$.

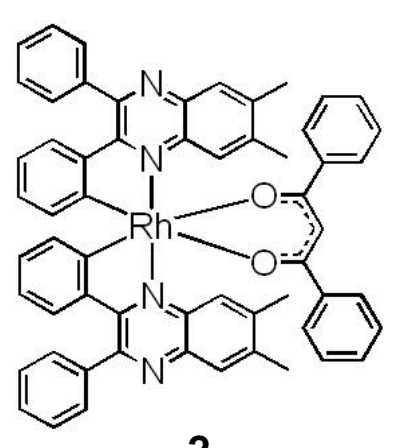

2

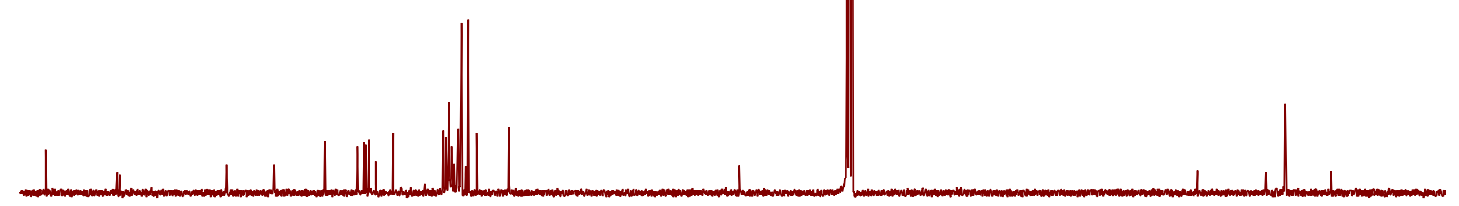

$\begin{array}{lllllllllllllllllll}180 & 170 & 160 & 150 & 140 & 130 & 120 & 110 & 100 \begin{array}{l}90 \\ \mathrm{fl}(\mathrm{ppm})\end{array} & 80 & 70 & 60 & 50 & 40 & 30 & 20 & 10 & 0\end{array}$

Figure S4. ${ }^{13} \mathrm{C}$ NMR spectrum of 2 in $\mathrm{CDCl}_{3}$ at $298 \mathrm{~K}$. 

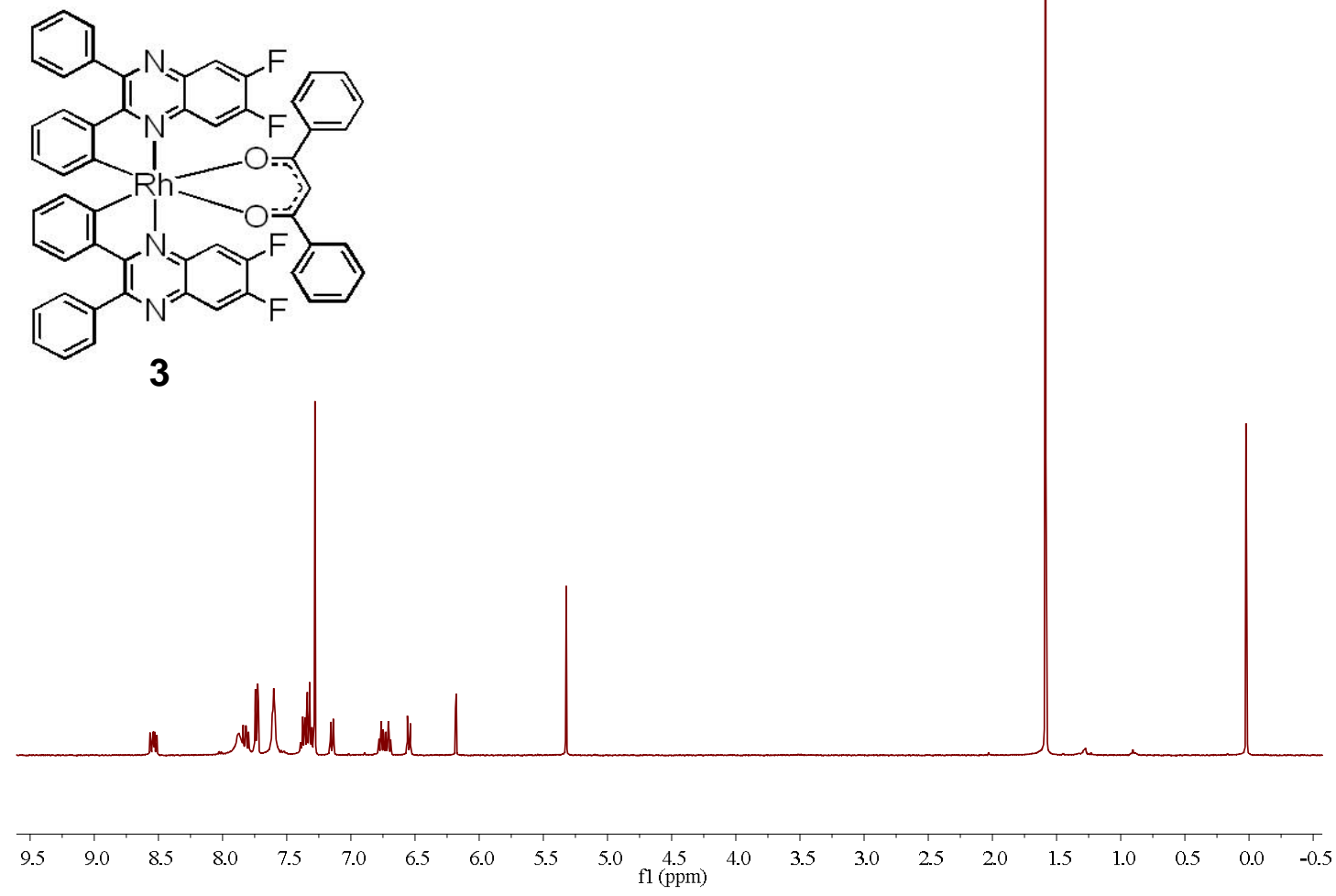

Figure S5. ${ }^{1} \mathrm{H}$ NMR spectrum of 3 in $\mathrm{CDCl}_{3}$ at $298 \mathrm{~K}$.

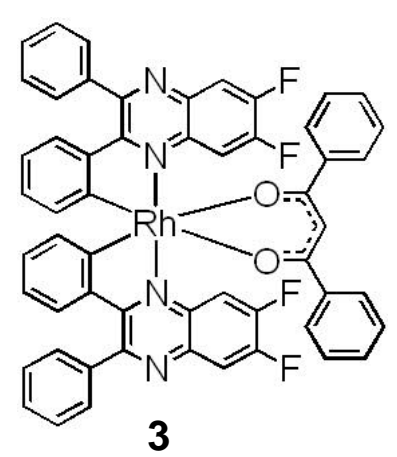

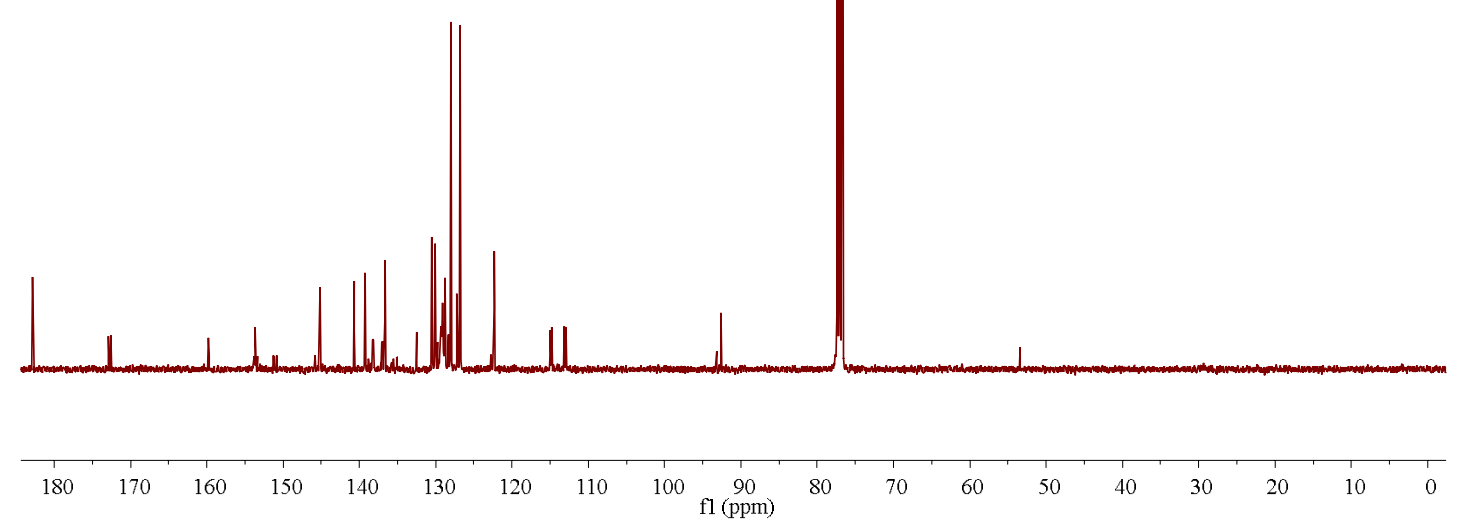

Figure S6. ${ }^{13} \mathrm{C}$ NMR spectrum of 3 in $\mathrm{CDCl}_{3}$ at $298 \mathrm{~K}$. 

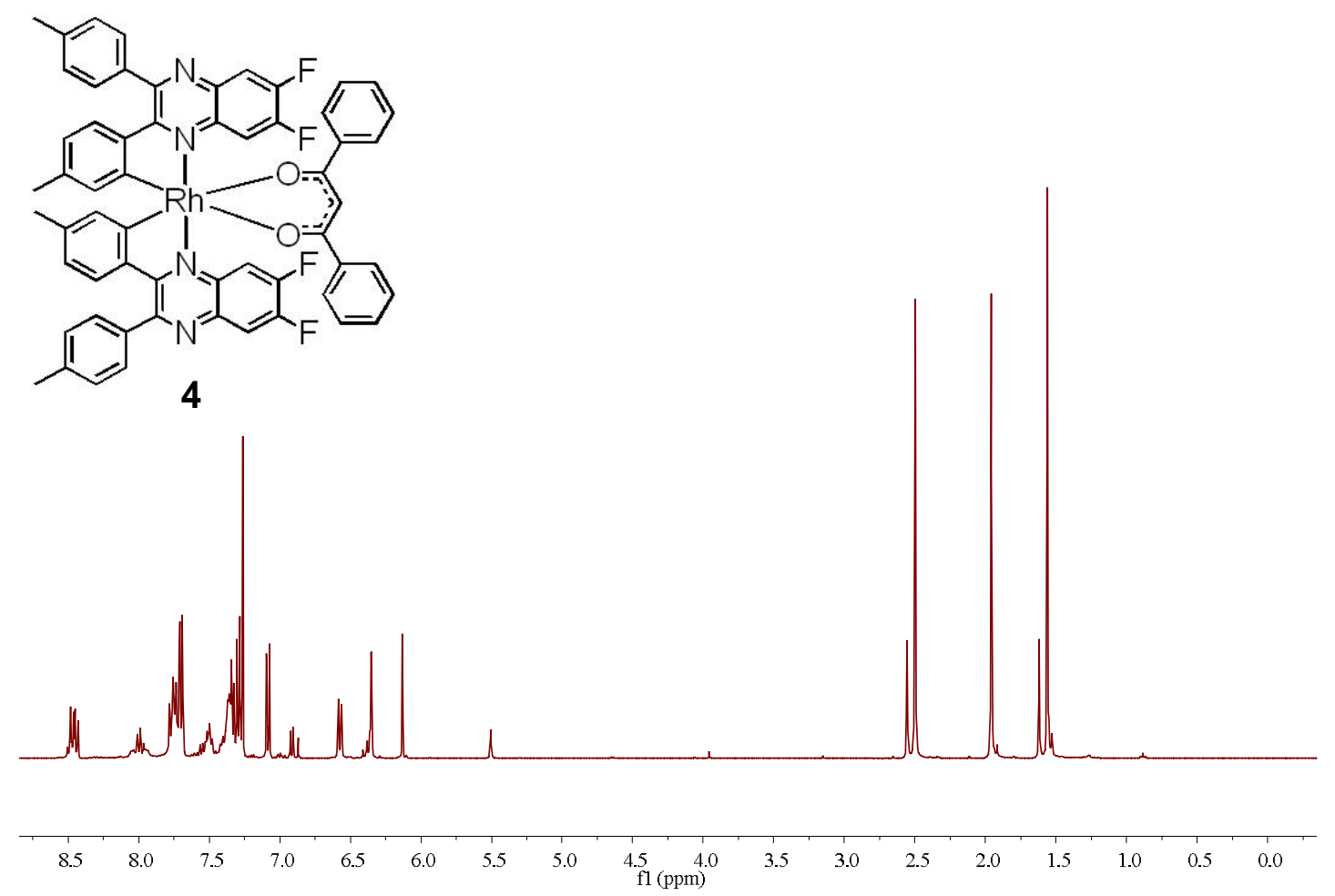

Figure S7. ${ }^{1} \mathrm{H}$ NMR spectrum of 4 in $\mathrm{CDCl}_{3}$ at $298 \mathrm{~K}$.

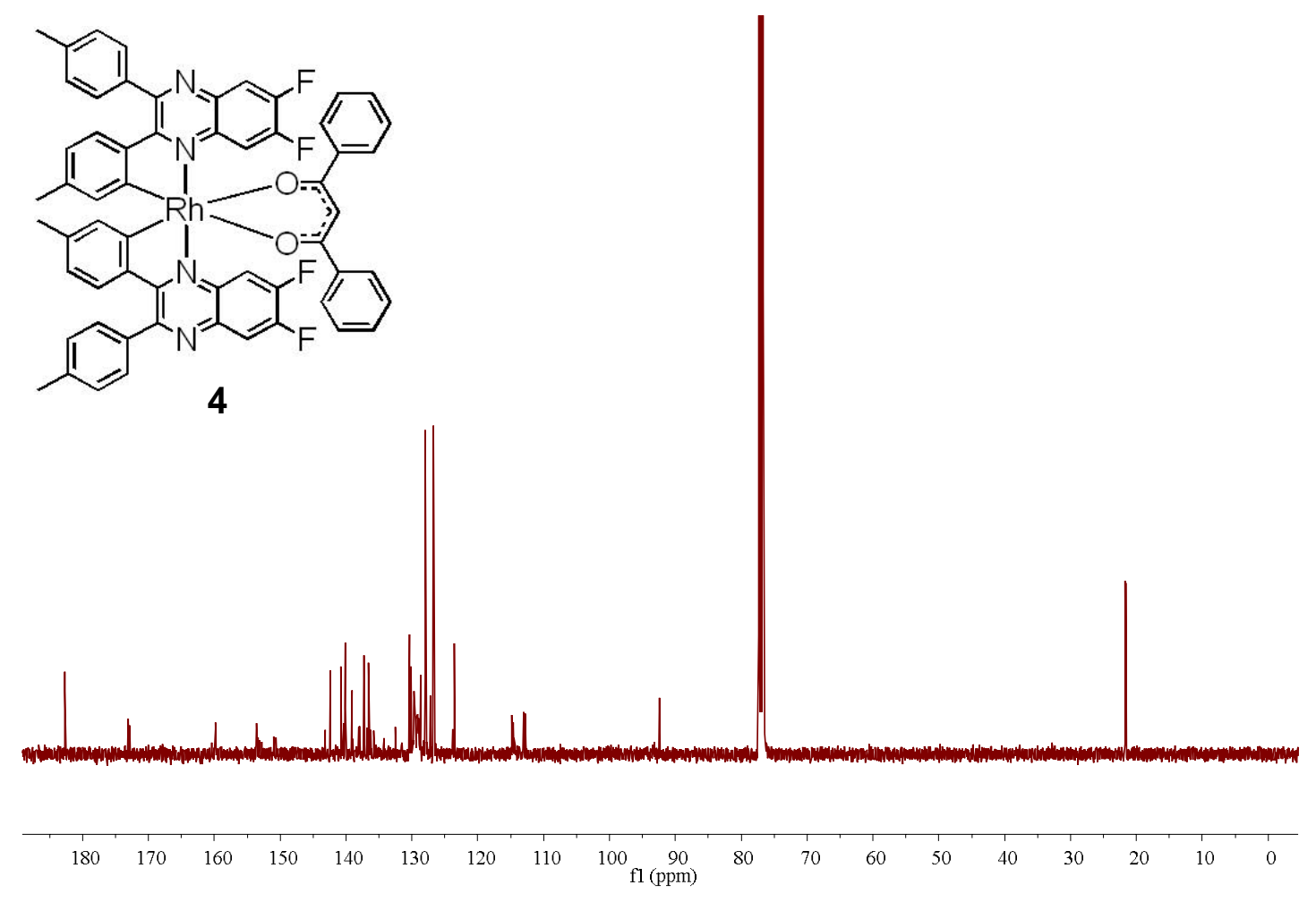

Figure S8. ${ }^{13} \mathrm{C}$ NMR spectrum of 4 in $\mathrm{CDCl}_{3}$ at $298 \mathrm{~K}$. 


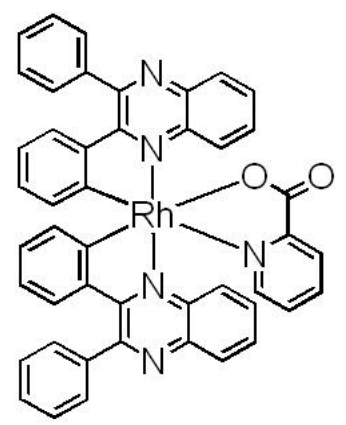

5
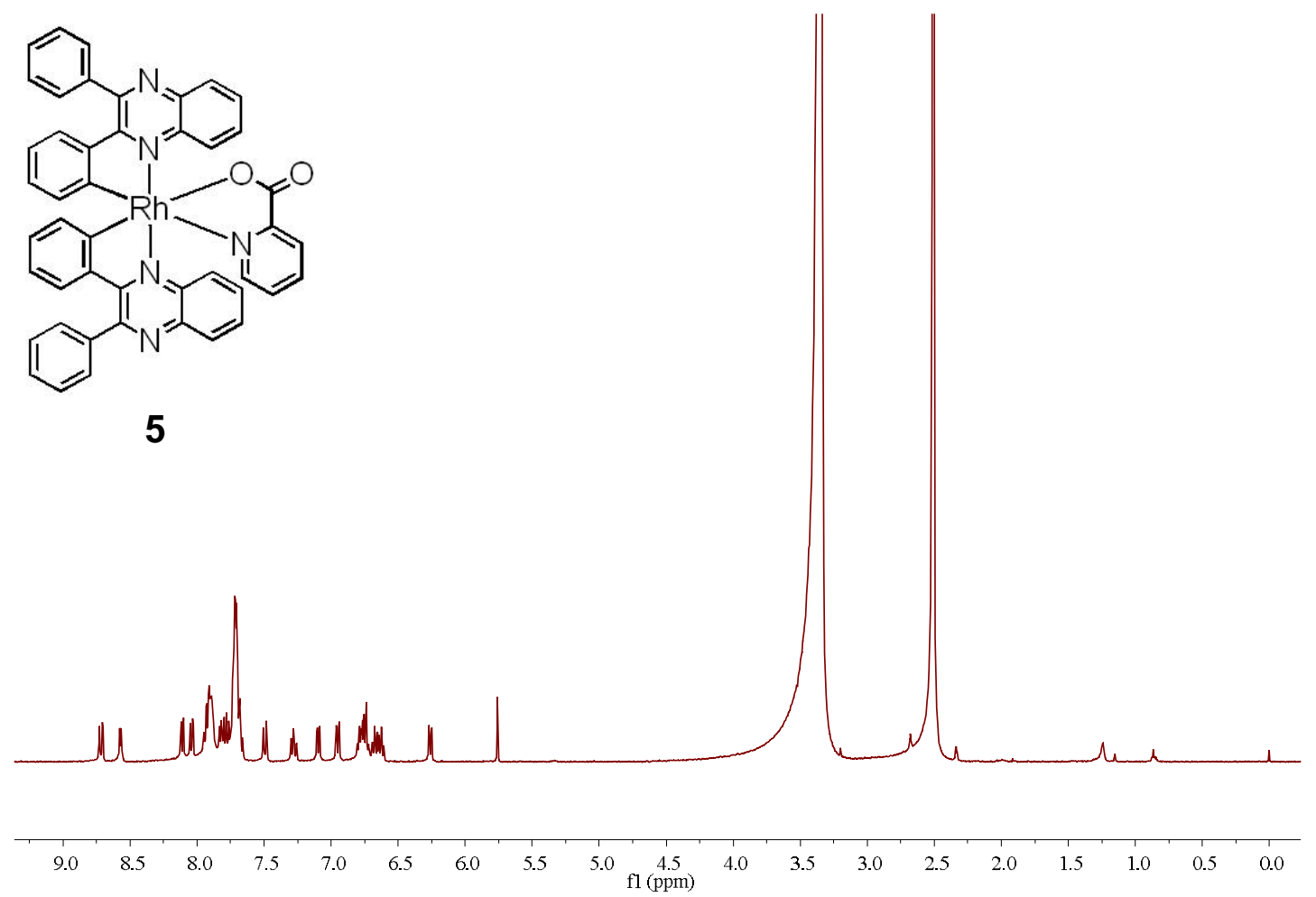

Figure S9. ${ }^{1} \mathrm{H}$ NMR spectrum of 5 in DMSO-d 6 at $298 \mathrm{~K}$.

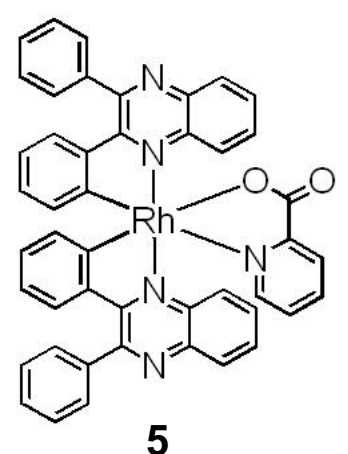

5

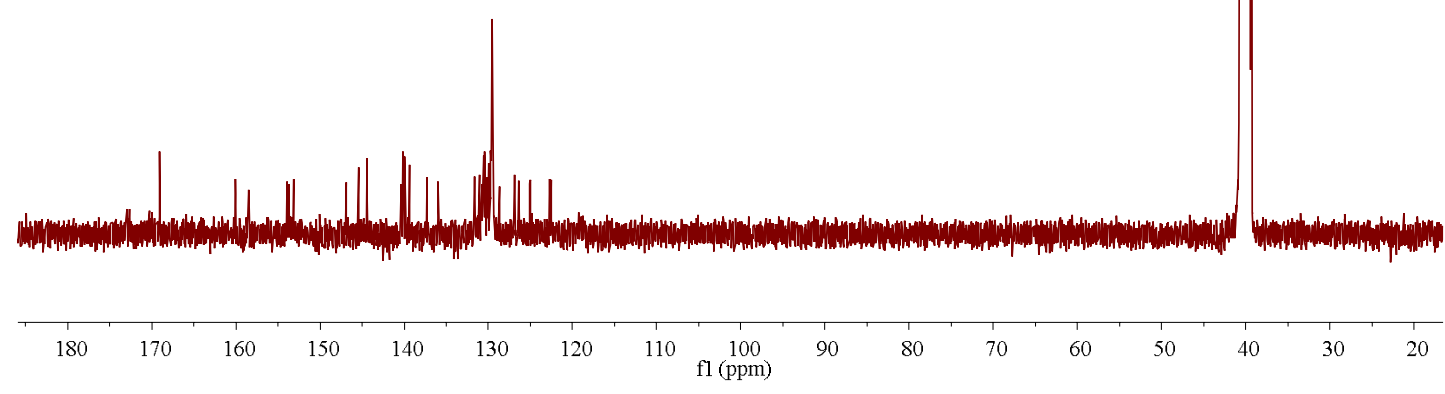

Figure S10. ${ }^{13} \mathrm{C}$ NMR spectrum of 5 in DMSO-d 6 at $298 \mathrm{~K}$. 


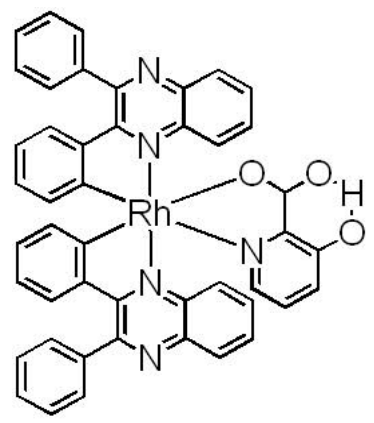

6

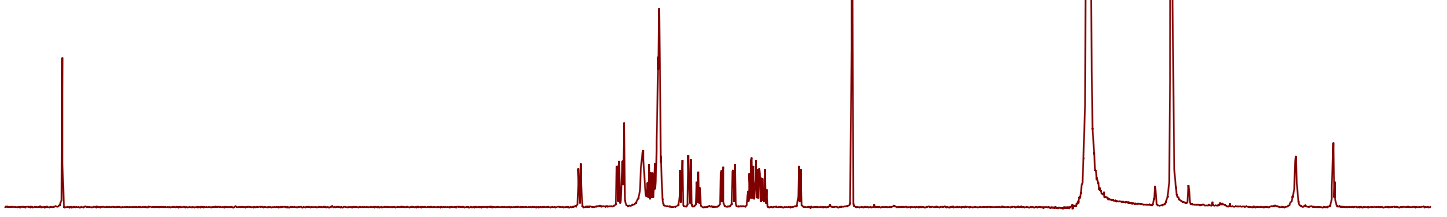

$\begin{array}{lllllllllllllllllllllllllllllll}13.5 & 12.5 & 11.5 & 10.5 & 9.5 & 9.0 & 8.5 & 8.0 & 7.5 & 7.0 & 6.5 & 6.0 & 5.5 & 5.0 & 4.5 & 4.0 & 3.5 & 3.0 & 2.5 & 2.0 & 1.5 & 1.0 & 0.5 & 0.0\end{array}$

Figure S11. ${ }^{1} \mathrm{H}$ NMR spectrum of 6 in DMSO-d 6 at $298 \mathrm{~K}$.

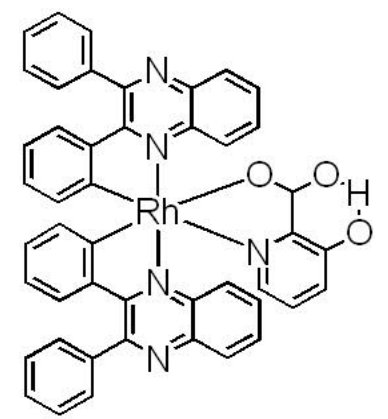

6

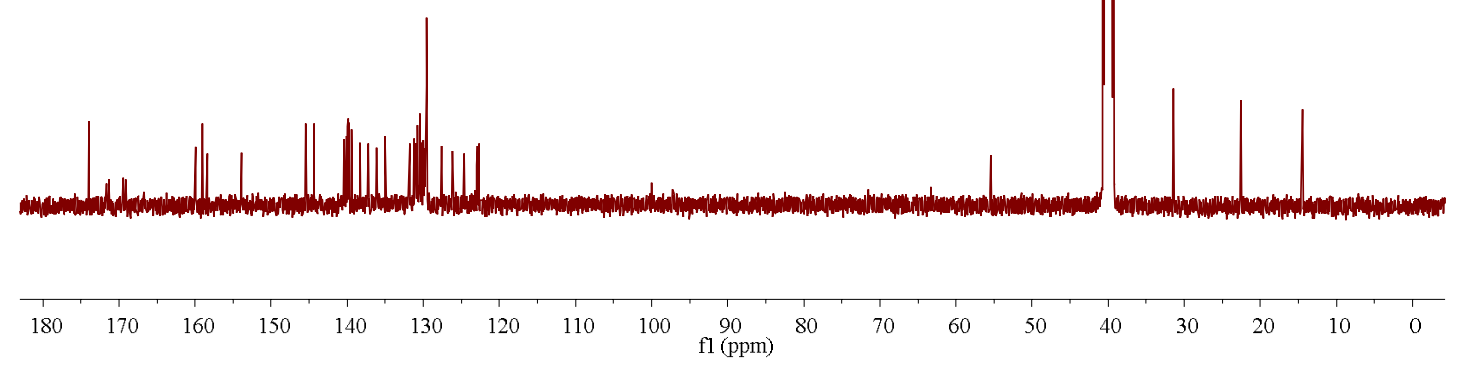

Figure $\mathrm{S} 12 .{ }^{13} \mathrm{C}$ NMR spectrum of 6 in DMSO-d 6 at $298 \mathrm{~K}$. 
1\#3-78 RT: 0.03-0.89 AV: 76 NL: $7.40 \mathrm{E} 5$

T: FTMS + p ESIFull ms [500.0000-1200.0000]

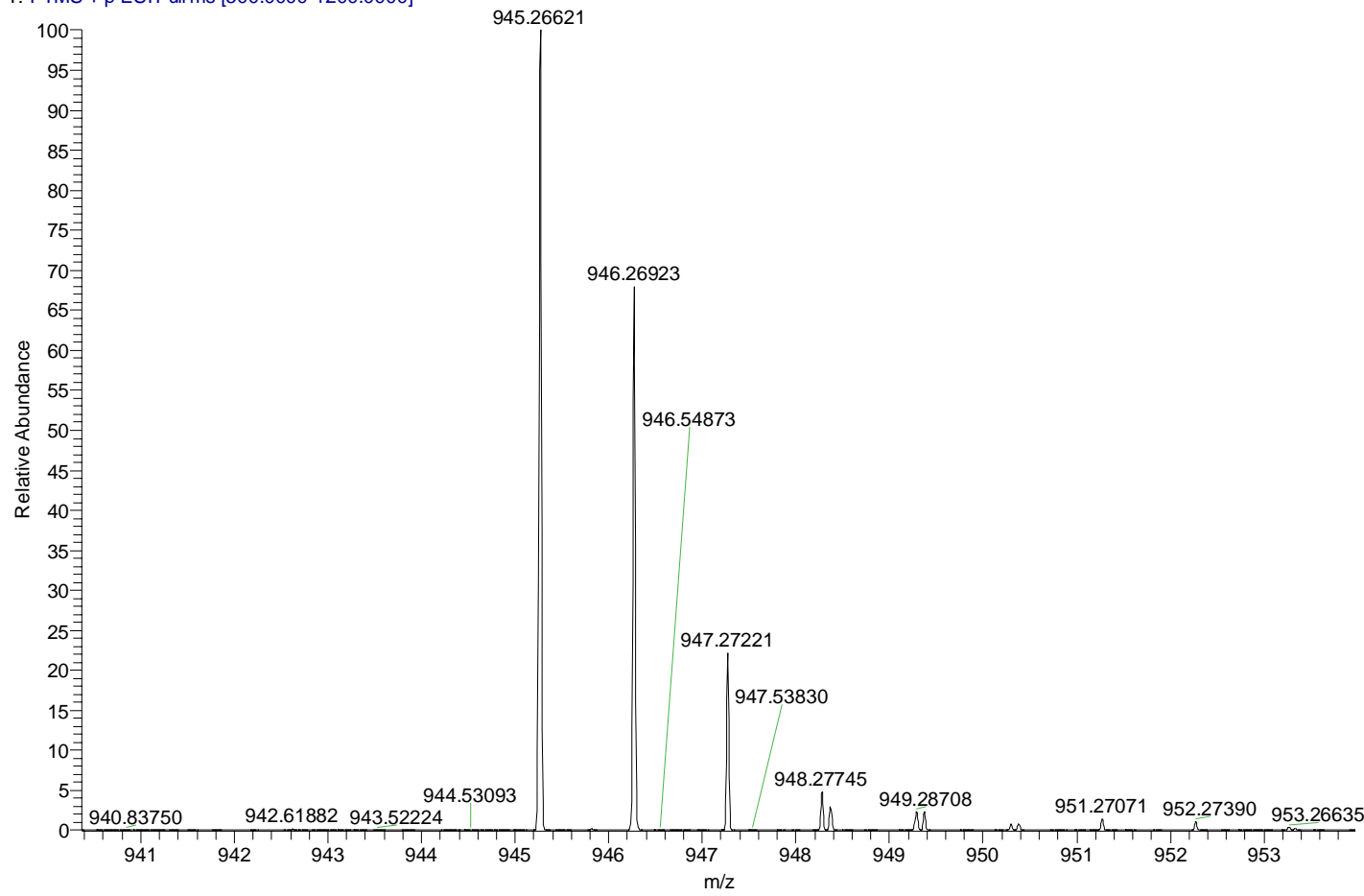

Figure S13. High resolution mass spectrum of $\mathbf{1}$.

2 \#2-48 RT: 0.02-0.54 AV: 47 NL: 2.26E6

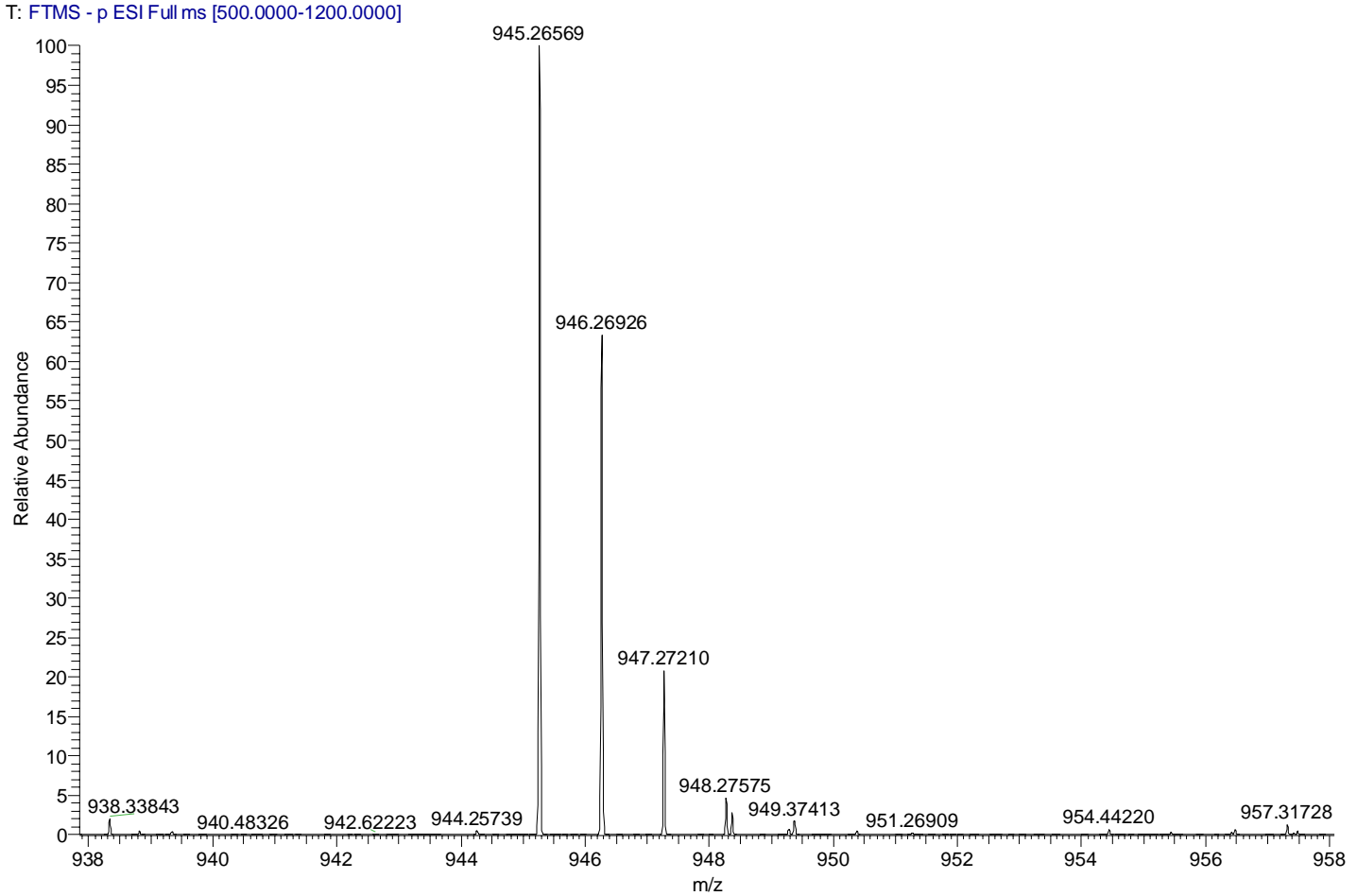

Figure S14. High resolution mass spectrum of 2. 
3\#1-77 RT: 0.01-0.88 AV: 77 NL: 1.33E6

T: FTMS + p ESI Full ms [500.0000-1200.0000]

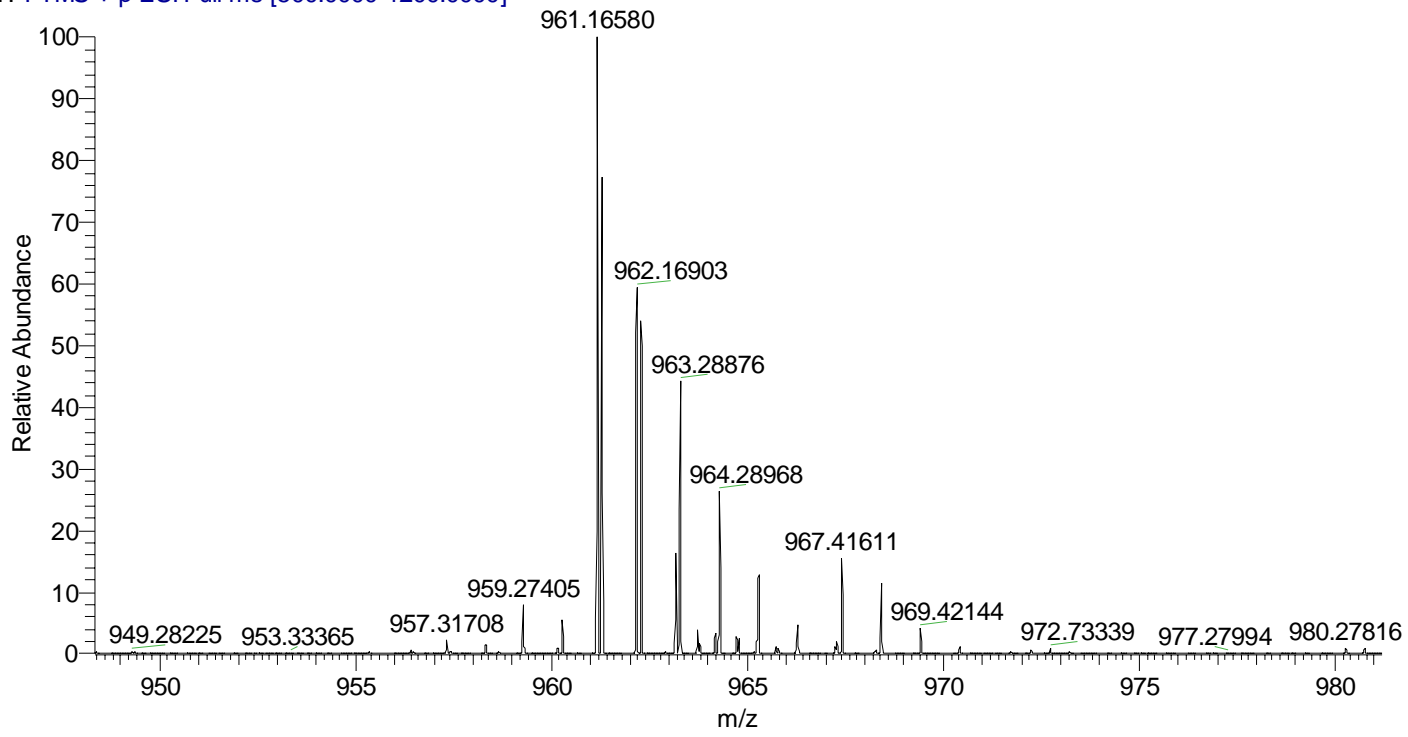

Figure S15. High resolution mass spectrum of $\mathbf{3}$.

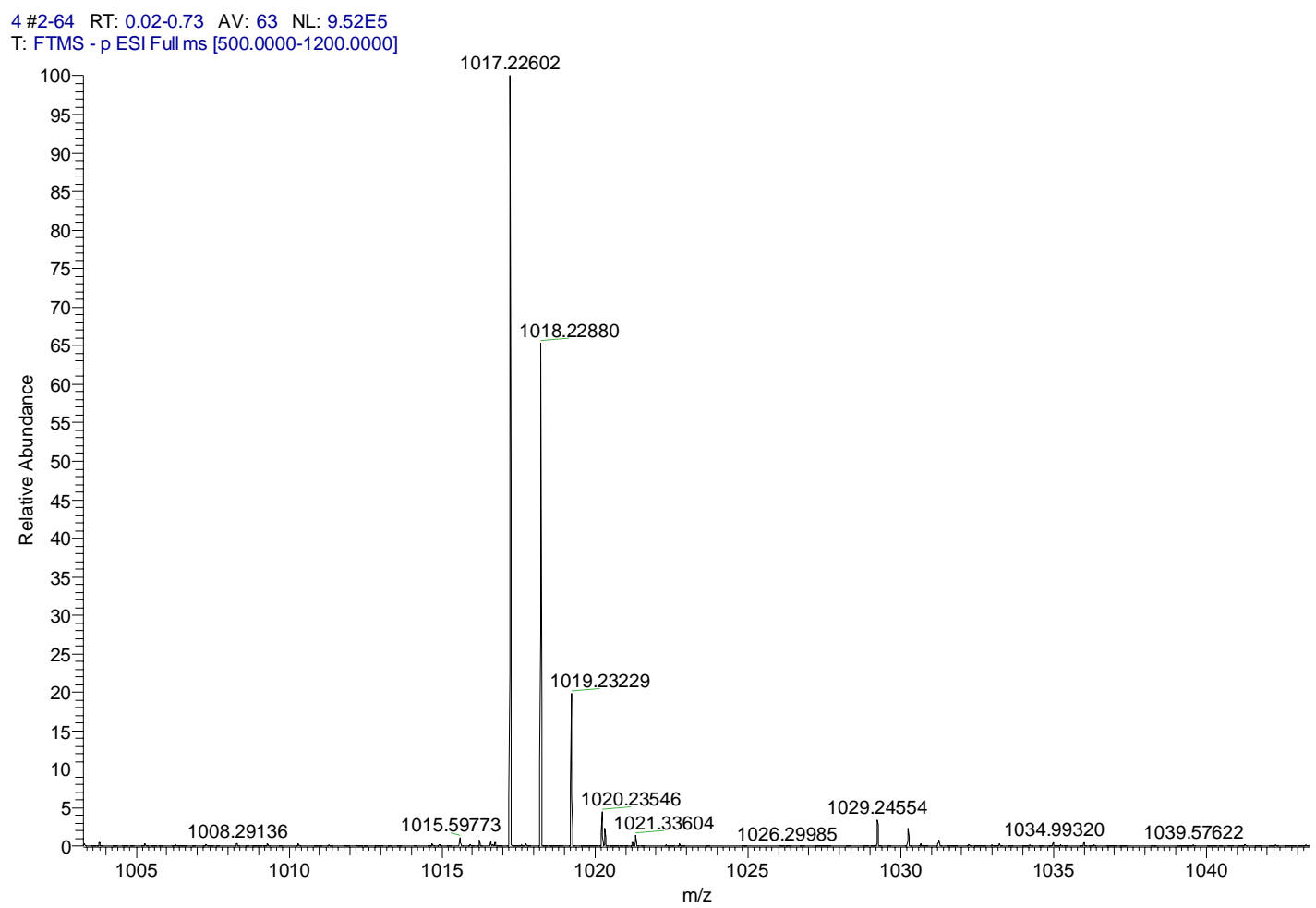

Figure S16. High resolution mass spectrum of 4 . 
5 \#1-38 RT: 0.01-0.43 AV: 38 NL: 8.09E7

T: FTMS + p ESI Full ms [500.0000-1200.0000]

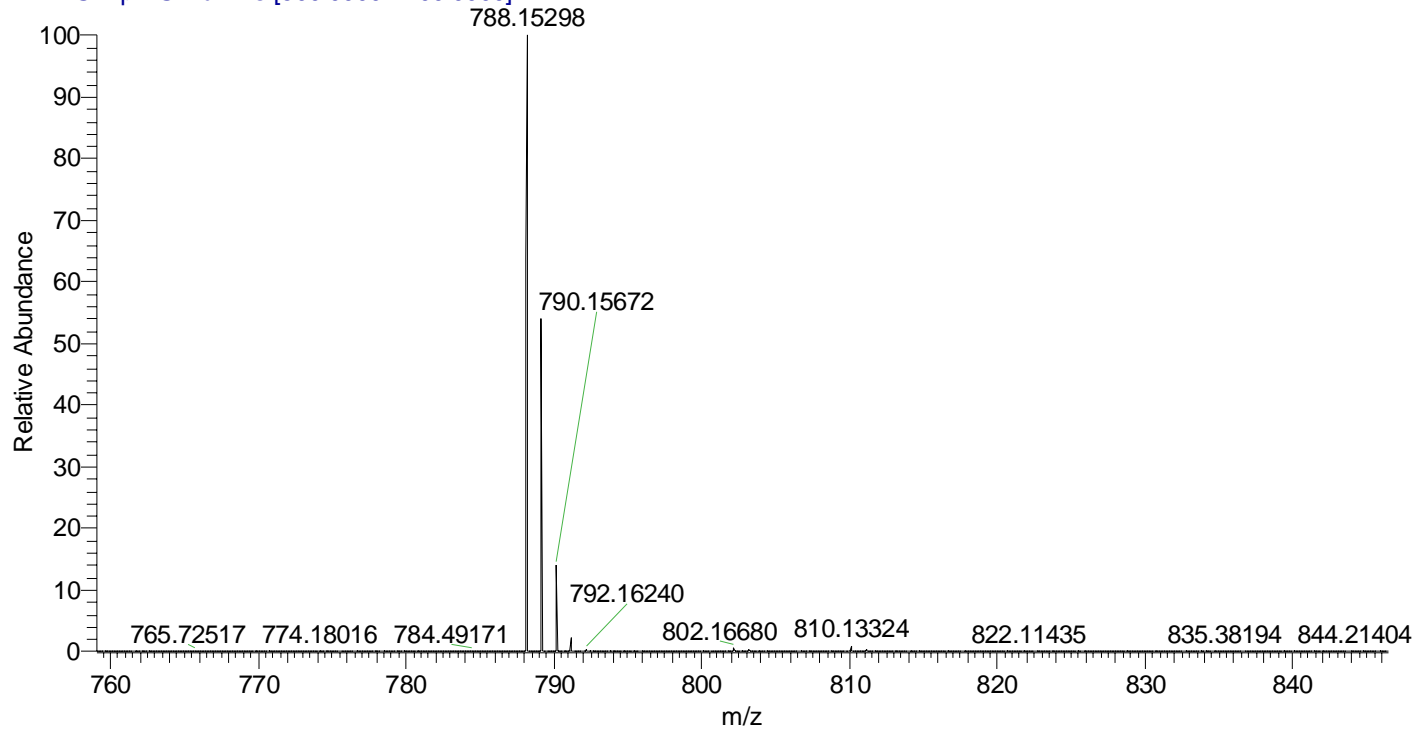

Figure S17. High resolution mass spectrum of 5.

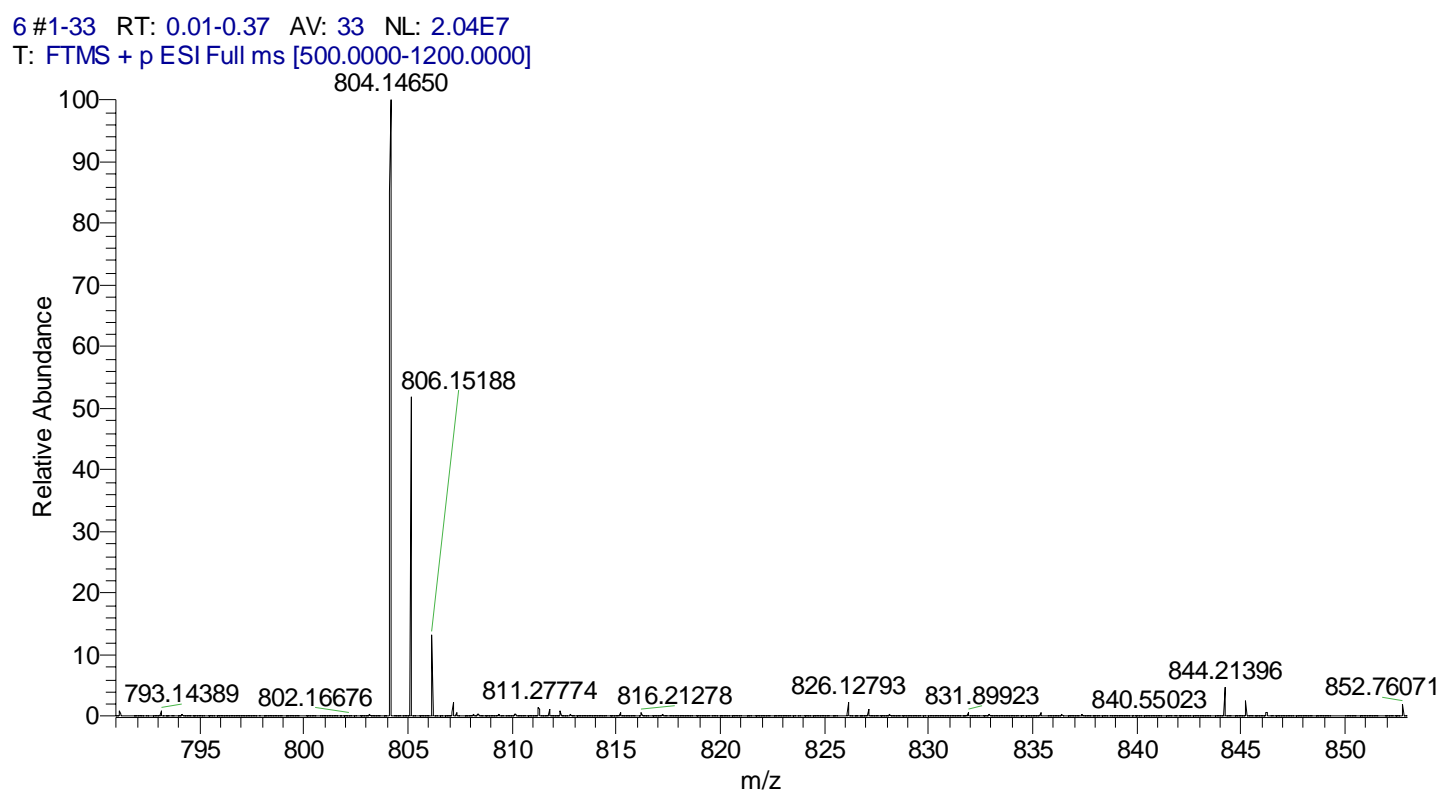

Figure S18. High resolution mass spectrum of 6. 

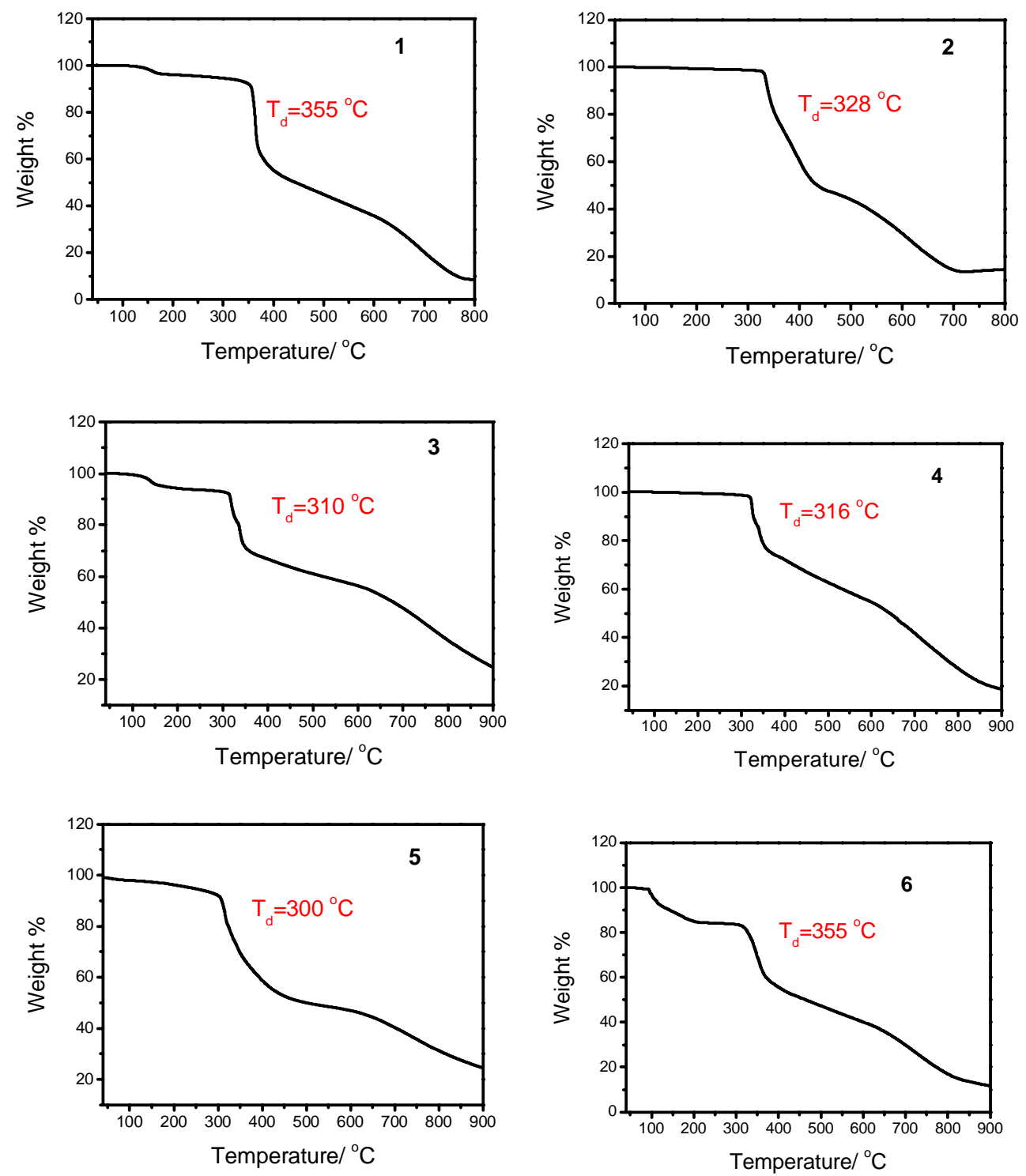

Figure S19. Thermogravimetric curves of 1-6. 

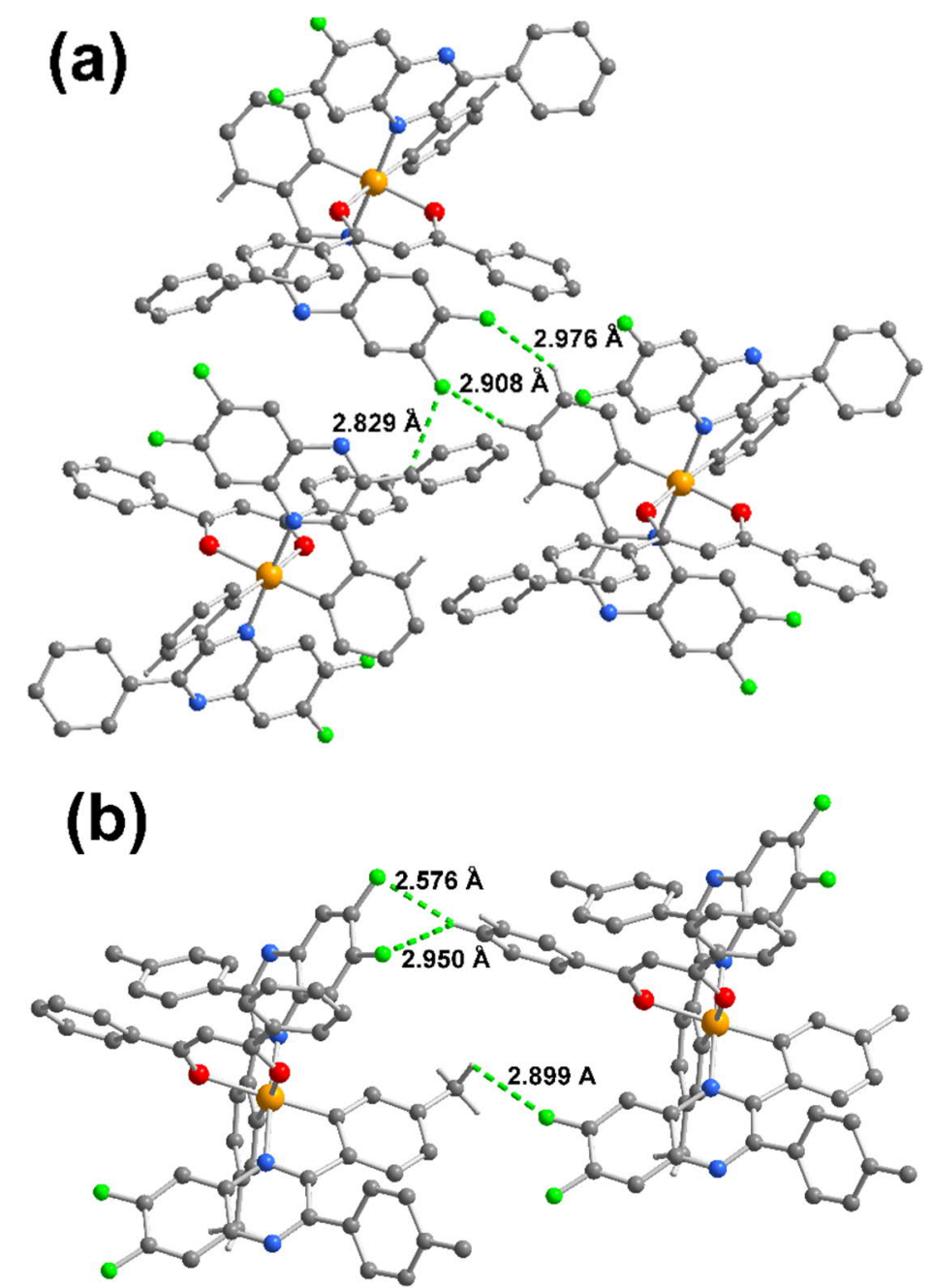

Figure S20. Crystal packings of $3(a)$ and 4 (b) with $\mathrm{C}-\mathrm{H} \cdots \mathrm{F}$ interactions with adjacent molecules. 

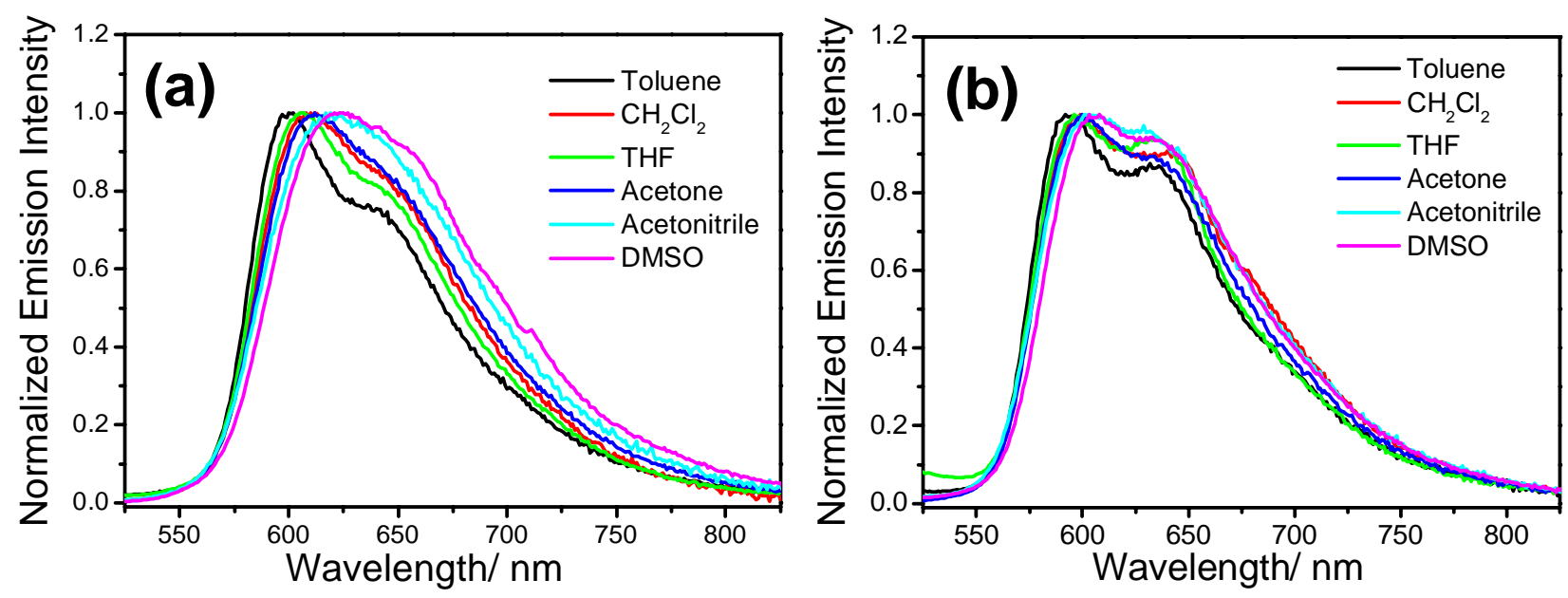

Figure S21. Emission spectra of $\mathbf{3}(\mathrm{a})$ and $\mathbf{5}$ (b) in different solvents with different polarity at $298 \mathrm{~K}$. 

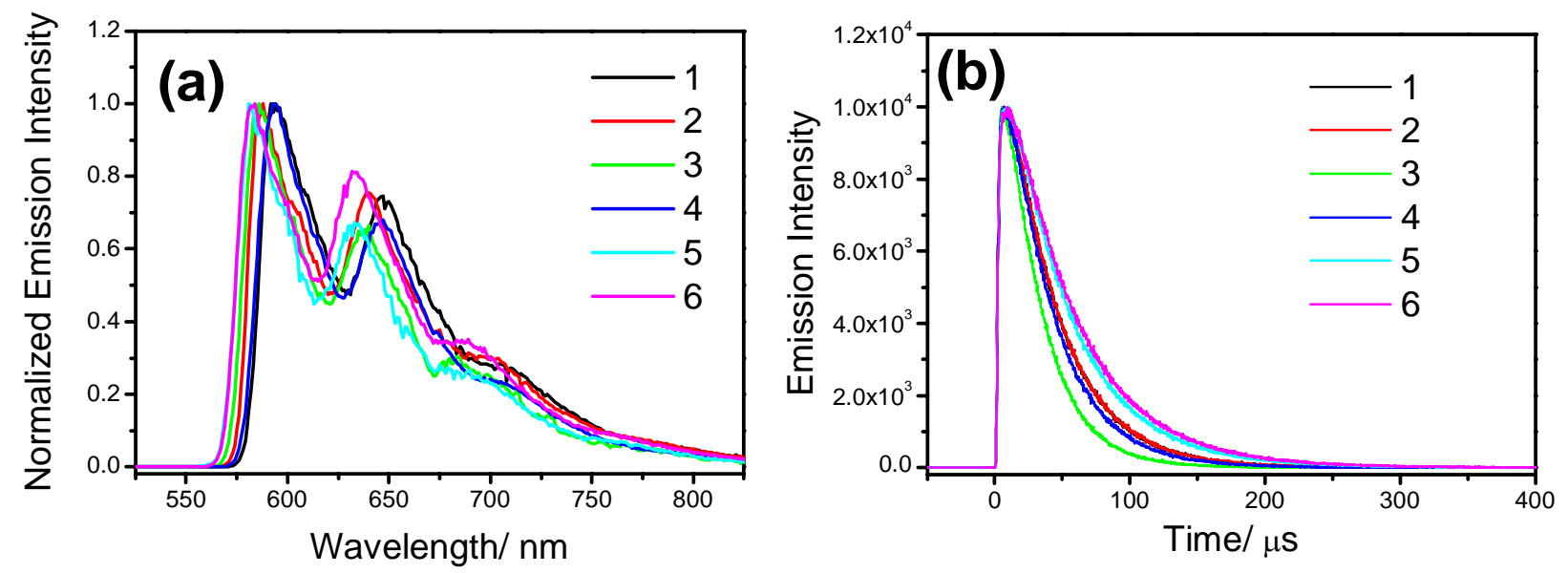

Figure S22. Normalized emission spectra (a) and the corresponding emission decay traces (b) of complexes 1-6 in 2-MeTHF at 77K. 

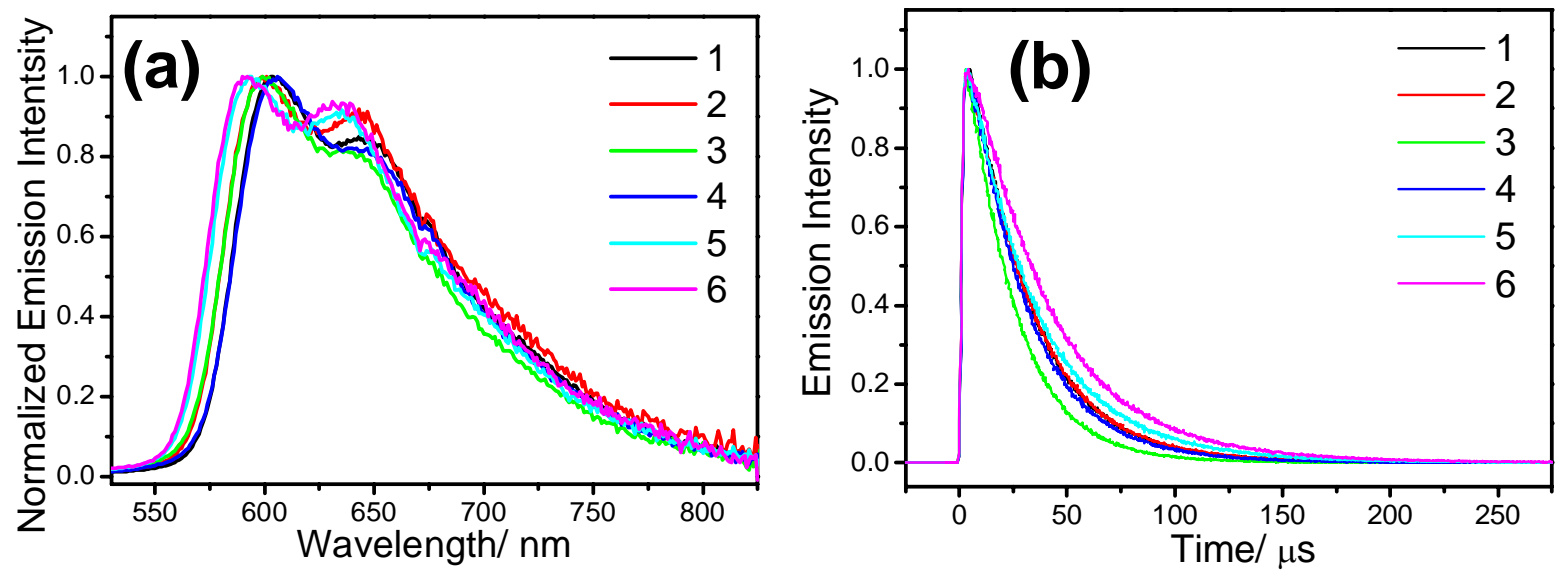

Figure S23. Normalized emission spectra (a) and the corresponding emission decay traces (b) of complexes 1-6 (2\% wt) in MCP thin film at $298 \mathrm{~K}$. 

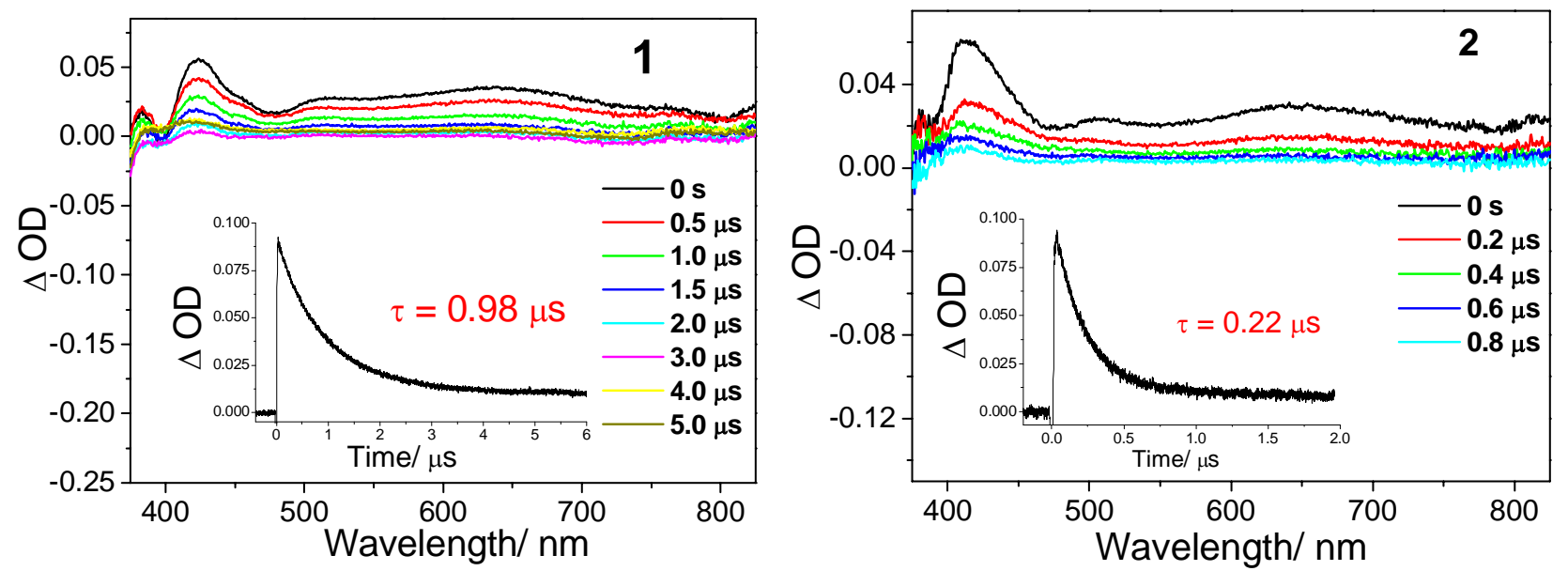

Figure S24. Transient absorption spectra of 1 and 2 (insets show the decay trace) in degassed $\mathrm{CH}_{2} \mathrm{Cl}_{2}$ at room temperature. 

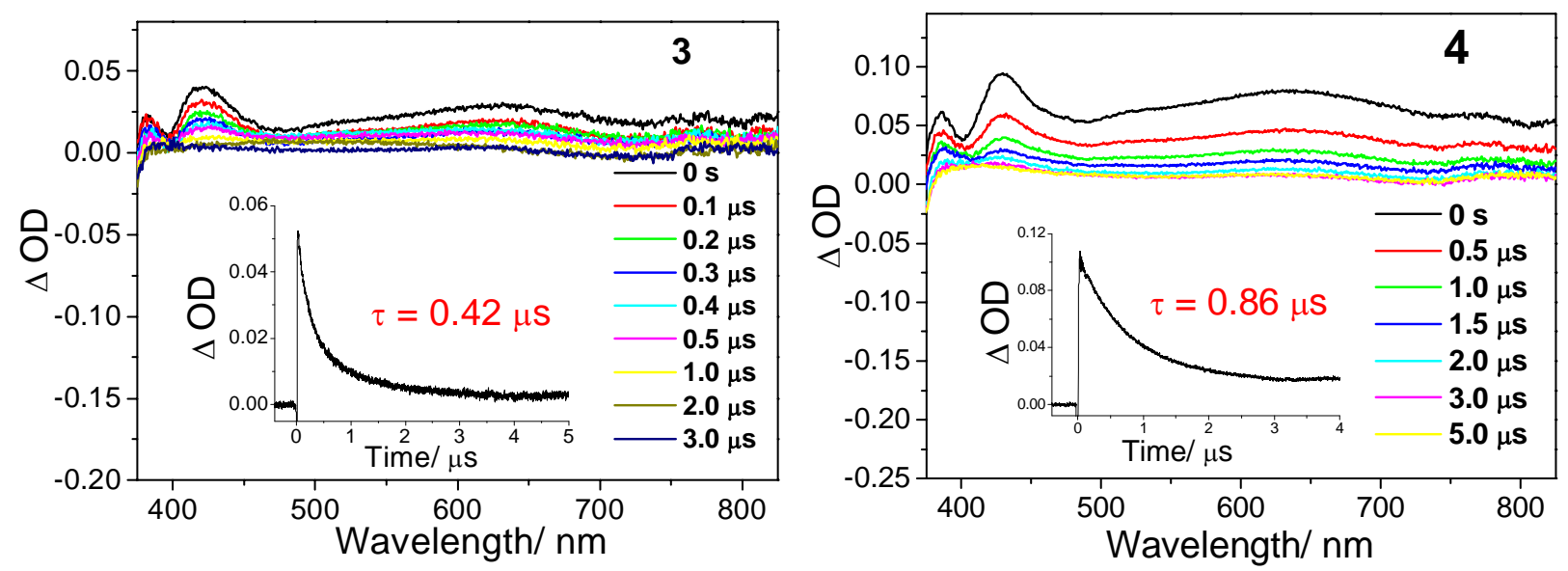

Figure S25. Transient absorption spectra of $\mathbf{3}$ and $\mathbf{4}$ (insets show the decay trace) in degassed $\mathrm{CH}_{2} \mathrm{Cl}_{2}$ at room temperature. 

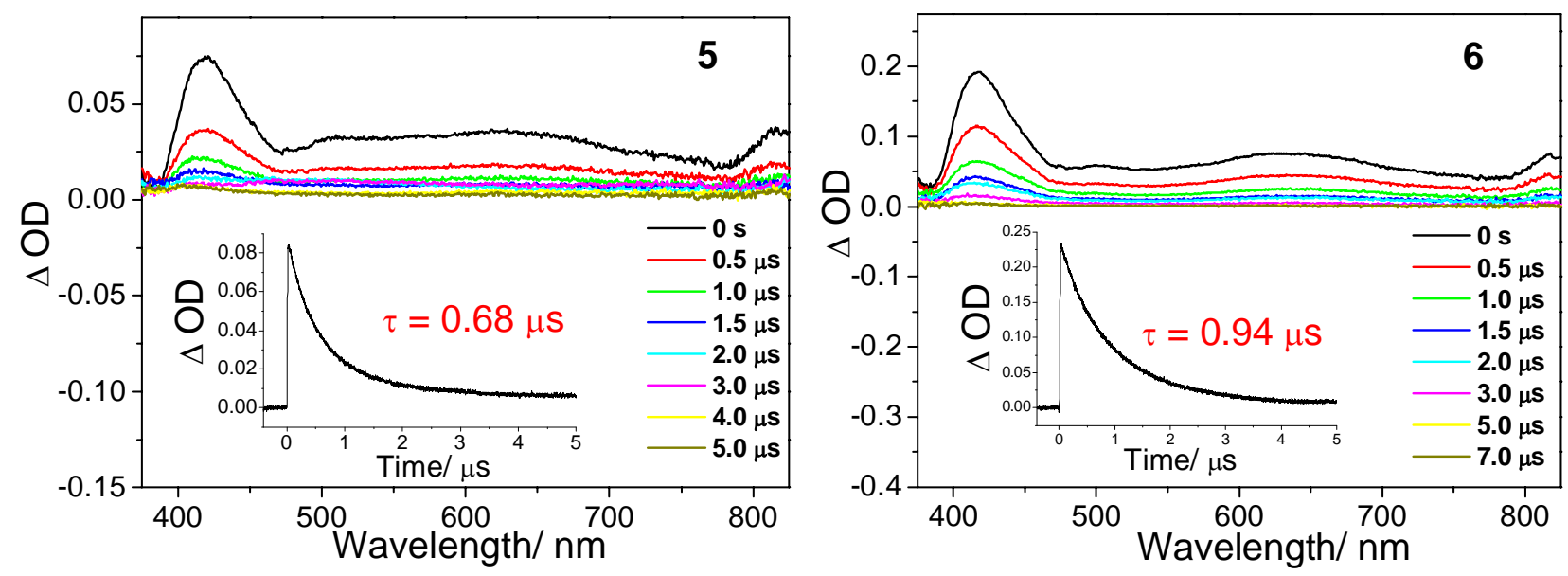

Figure S26. Transient absorption spectra of $\mathbf{5}$ and $\mathbf{6}$ (insets show the decay trace) in degassed $\mathrm{CH}_{2} \mathrm{Cl}_{2}$ at room temperature. 


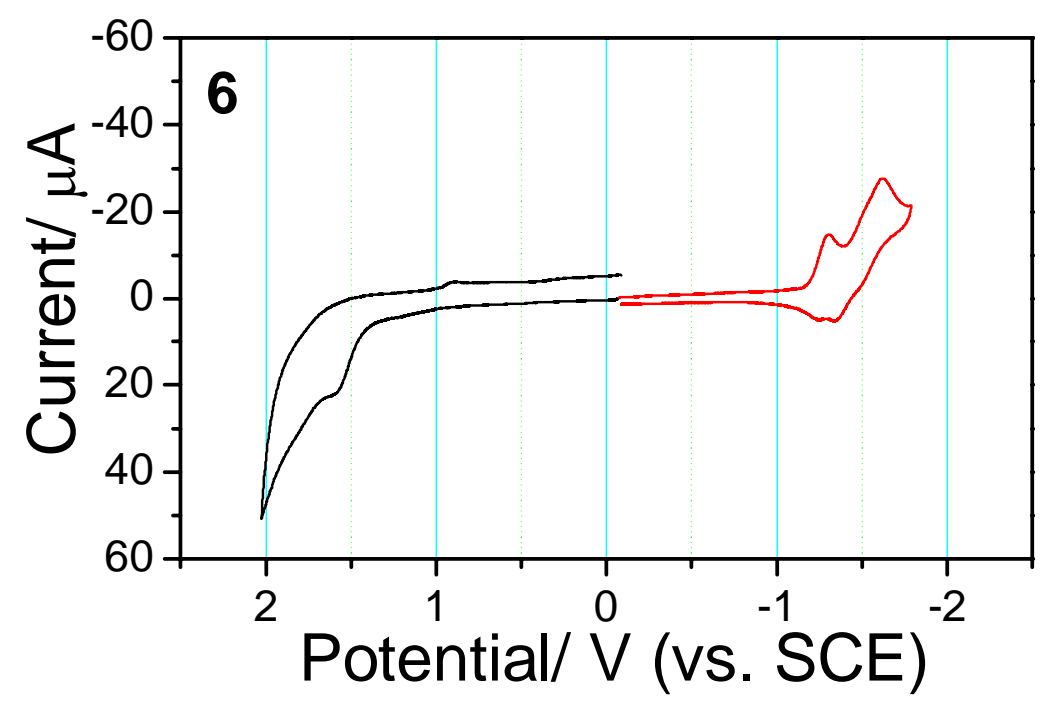

Figure S27. Cyclic voltammogram of 6 in dichloromethane $\left(0.1 \mathrm{M}^{\mathrm{n} B \mathrm{~B}_{4}} \mathrm{NPF}_{6}\right)$. 


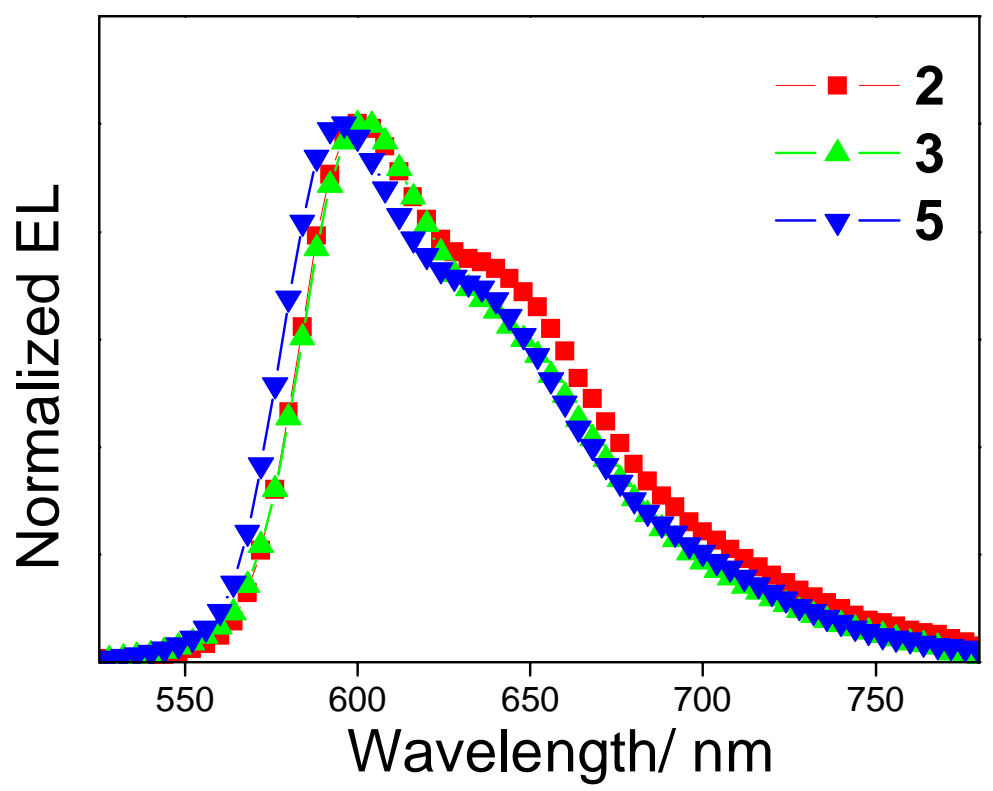

Figure S28. Normalized EL spectra of solution-processed devices based on $10 \mathrm{wt} \% \mathbf{2}, \mathbf{3}$ and 5 at a luminance of $100 \mathrm{~cd} \mathrm{~m}^{-2}$. 

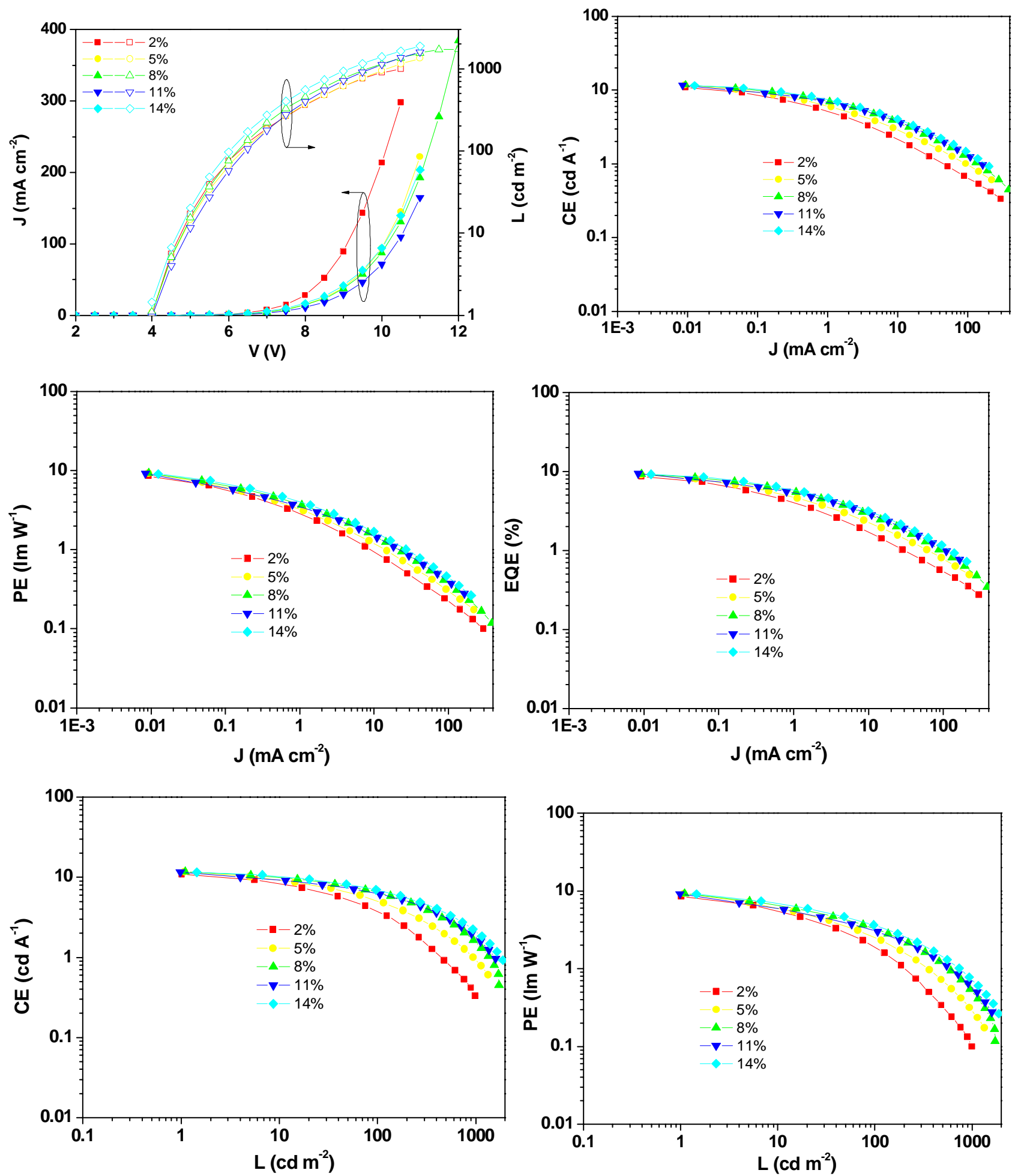

Figure S29. EL characteristics based on 2 with device structure of ITO/a-NPD (40nm)/TCTA (5nm)/x\%2:mCBP(20nm)/Tm3PyP26PyB(50nm)/LiF(1nm)/Al(150n $\mathrm{m})$, where Tm3PyP26PyB = 1,3,5-tris(6-(3-(pyridin-3-yl)phenyl)pyridine-2-yl)benzene. 

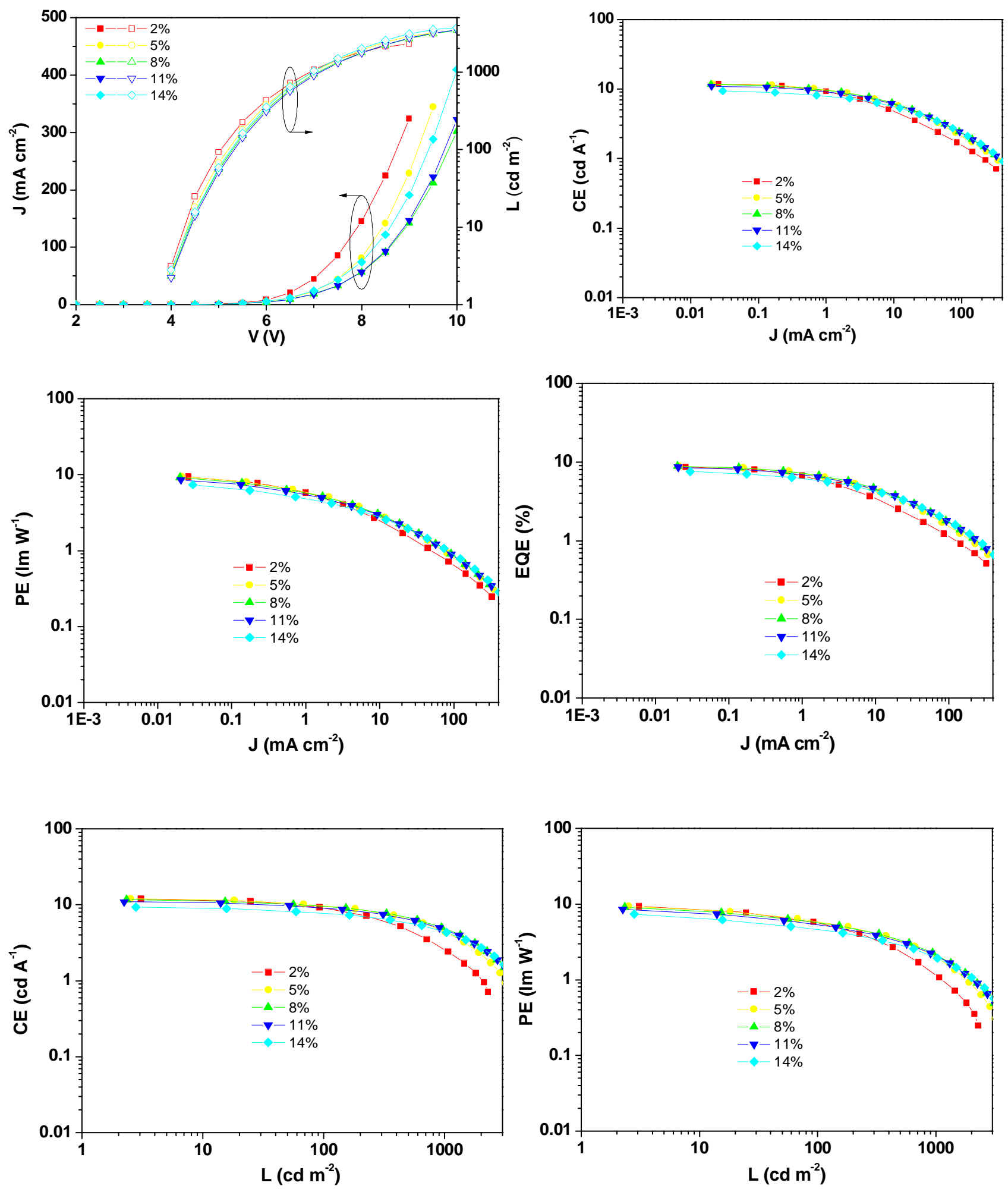

Figure S30. EL characteristics based on 3 with device structure of ITO/a-NPD (40nm)/TCTA (5nm)/x\%3:mCBP(20nm)/Tm3PyP26PyB(50nm)/LiF(1nm)/Al(150n $\mathrm{m})$, where Tm3PyP26PyB = 1,3,5-tris(6-(3-(pyridin-3-yl)phenyl)pyridine-2-yl)benzene. 